\title{
B cell repertoire expansion occurs in meningeal ectopic lymphoid tissue
}

\author{
Klaus Lehmann-Horn, ${ }^{1,2}$ Sheng-zhi Wang, ${ }^{1}$ Sharon A. Sagan, ${ }^{1,2}$ Scott S. Zamvil, ${ }^{1,2}$ \\ and H.-Christian von Büdingen ${ }^{1,3}$ \\ 'Department of Neurology, ${ }^{2}$ Program in Immunology, ${ }^{3}$ Program in Biomedical Sciences, UCSF, San Francisco, \\ California, USA.
}

\begin{abstract}
Ectopic lymphoid tissues (ELT) can be found in multiple sclerosis (MS) and other organspecific inflammatory conditions. Whether ELT in the meninges of central nervous system (CNS) autoimmune disease exhibit local germinal center (CC) activity remains unknown. In an experimental autoimmune encephalomyelitis model of CNS autoimmunity, we found activationinduced cytidine deaminase, a GC-defining enzyme, in meningeal ELT (mELT) densely populated by $B$ and T cells. To determine GC activity in mELT, we excised meningeal lymphoid aggregates using laser capture microscopy and evaluated B cell repertoires in mELT and secondary lymphoid organs by next-generation immune repertoire sequencing. We found immunoglobulin heavy chain variable region sequences that were unique to $\mathrm{mELT}$ and had accumulated functionally relevant somatic mutations, together indicating localized antigen-driven affinity maturation. Our results suggest that B cells in mELT actively participate in CNS autoimmunity, which may be relevant to mELT in MS and ELT in other chronic inflammatory conditions.
\end{abstract}

Authorship note: K. Lehmann-Horn and $S$. Wang are co-first authors.

Conflict of interest: The authors have declared that no conflict of interest exists.

Submitted: February 23, 2016 Accepted: October 25, 2016 Published: December 8, 2016

Reference information: JCI Insight. 2016;1(20):e87234. doi:10.1172/jii.insight.87234.

\section{Introduction}

Ectopic (or tertiary) lymphoid tissue (ELT) can be found in the target organs of infectious and autoimmune diseases, cancer, and transplant rejection (1). Like secondary lymphoid tissue, ELT contain germinal centers (GCs), where B cells proliferate, and undergo somatic hypermutation (SHM) of their rearranged immunoglobulin (Ig) genes for antigen-driven affinity maturation and class switch recombination (CSR) $(1,2)$. In infectious disease, ELT are believed to contribute to pathogen control. In autoimmunity however, ELT may contribute to local immune activation and promote tissue destruction via autoantibody production, complement activation, and release of proinflammatory cytokines.

Expression of activation-induced cytidine deaminase (AICDA) by B cells is required for SHM and CSR and hence for GC reaction. Presence of AICDA was previously demonstrated in ELT in patients with autoimmune conditions, including rheumatoid arthritis (3), Sjögren's disease (4), and secondary progressive multiple sclerosis (SPMS) (5), suggesting that GC activity is a principal feature of ELT. Centroblasts, lymphotoxin- $\alpha$, CXC ligand 12 (CXCL12), and CXCL13, key factors in lymphoid neogenesis, are present in the CSF of MS patients (6). Prior to the advent of next-generation sequencing, a number of studies found evidence for local SHM in ELT by investigating immunoglobulin genes expressed by a limited selection of ectopic B cells using Sanger sequencing (7-11). However, a comprehensive analysis of the B cell repertoire, enabling discrimination between migration of secondary lymphoid organ-derived (SLO-derived) antigen-specific B cell clones, and in situ B cell receptor (BCR) maturation in ELT is currently lacking.

MS is a common inflammatory disease of the CNS. After an initial relapsing-remitting phase, many patients enter a secondary progressive phase, which is characterized by fewer or no attacks but by insidious worsening of neurological deficits. B cell-rich lymphoid follicle-like tissues have been described in the meninges in SPMS (12-16). Meningeal B cell aggregates in spontaneous experimental autoimmune encephalomyelitis (EAE) bear a striking resemblance to those found in SPMS (17-21). Together, histologic and morphologic studies of meningeal inflammatory infiltrates in SPMS and EAE have provided evidence suggesting these tissues contain GCs and may represent ectopic lymphoid follicle-like structures; to date, this has not been formally demonstrated.

Our goal in this study was to evaluate whether B cell repertoire diversification occurs in situ in meningeal B cell aggregates. We used a spontaneous EAE model that is associated with meningeal B cell aggre- 
gates (20-22). All B cells in these mice contain a rearranged myelin oligodendrocyte glycoprotein-specific (MOG-specific) BCR heavy chain (IgH) gene, which served as a template to examine SHM in various immune compartments, including meningeal ELT (mELT). We detected AICDA by immunohistochemistry and qPCR. Immune repertoire sequencing (RepSeq) of immunoglobulin heavy chain variable regions (Ig-VH) revealed evidence for local antigen-driven SHM and CSR in mELT. We cloned mutated IgGVH genes found exclusively in mELT, expressed the corresponding recombinant monoclonal antibodies $(\mathrm{rmAb})$, and evaluated their binding affinities for MOG. Our results demonstrate that affinity maturation occurs in B cells within mELT and thus unequivocally establish mELT as sites of local GC activity in CNS autoimmunity.

\section{Results}

mELT in Th $\times 2 D 2$ mice are rich in $B$ cells and contain AICDA. $\mathrm{MOG}_{35-55}$-reactive $\mathrm{T}$ cell receptor (TCR) transgenic (2D2, TCR ${ }^{\mathrm{MOG}}$ ) mice crossed with MOG Ig-VH knockin $\left(\mathrm{Th}, \mathrm{IgH}^{\mathrm{MOG}-\mathrm{ki}}\right)$ mice $(\mathrm{Th} \times 2 \mathrm{D} 2 \mathrm{mice})$ develop spontaneous chronic EAE (20-22) associated with abundant meningeal B cell-rich aggregates, particularly along the spinal cord $(20,21)$. We first evaluated histological characteristics of mELT and confirmed the presence of mostly B220 $0^{+}\left(\mathrm{CD} 45 \mathrm{R}^{+}\right) \mathrm{B}$ cells, but also $\mathrm{CD}^{+} \mathrm{T}$ cells (Figure $1, \mathrm{~A}$ and B), with zones resembling $\mathrm{B}$ and $\mathrm{T}$ cell zones in secondary lymphoid follicles. mELT frequently extended $>1 \mathrm{~mm}$ axially and were identified in all Th $\times 2 \mathrm{D} 2 \mathrm{EAE}$ mice studied here (Supplemental Table 1; supplemental material available online with this article; doi:10.1172/jci.insight.87234DS1). By immunohistochemistry, AICDA, an enzyme expressed during GC development, was detected in mELT (Figure 1C), but not in spinal cord parenchymal infiltrates. Similar to mELT, AICDA expression in the spleen was confined to single cells or small clusters of cells within follicular structures (Figure 1D). AICDA mRNA was detectable in spinal cord fragments (including the meninges) of $\mathrm{Th} \times 2 \mathrm{D} 2 \mathrm{EAE}$ mice (Figure $1 \mathrm{~F}$ ). On average, AICDA transcript levels were 11.6-fold higher in these spinal cords in comparison to a tissue without secondary or tertiary lymphoid structures (kidneys). No AICDA transcripts were detected in the spinal cords of healthy WT mice. As expected, high levels of AICDA mRNA were found in the spleen (Figure 1G).

$I g-V H$ repertoire analysis of $m E L T$ and peripheral immune compartments. Laser capture microscopy (LCM) was used to collect mELT from $5 \mathrm{Th} \times 2 \mathrm{D} 2$ mice with EAE (Supplemental Figure 1). From each mouse we combined 5-10 adjacent 20- $\mu \mathrm{m}$ sections of lymphocyte-rich meningeal tissue from at least one structurally contiguous mELT. We then performed next-generation IgM- and IgG-VH RepSeq of mELT and peripheral immune compartments to understand whether mELT are capable of supporting BCR affinity maturation and class switching. Our Ig PCR approach yielded sequence information for the VH region (complementarity determining region 1 [CDR1], framework region 2 [FR2], CDR2, FR3, CDR3), and alignment/comparison to the knockin germline Ig-VH derived from the 8.18-c5 anti-MOG antibody-producing hybridoma (23) permitted mutational analysis covering the Ig-VH portions most relevant for antigen binding. Furthermore, the location of the universal IgG constant region reverse primer yielded sufficient sequence information to determine whether an individual IgG-VH was expressed as IgG1, $-2 a / c,-2 b$, or -3 . A summary of the number of sequencing reads acquired from mELT, SLO, or blood from each mouse is shown in Table 1.

CSR occurs in mELT. Th $\times 2 \mathrm{D} 2$ mice produce increased amounts of anti-MOG IgG1 antibodies $(21,22)$. Interestingly, when comparing relative representation of IgG subclasses (IgG1, IgG2a/c, IgG2b, and IgG3) among our sequences from different tissues, we found most striking overrepresentation of IgG1 in mELT (Figure 2), suggesting either biased local CSR or preferential expansion of IgG1 B cells in mELT. We also found mELT-exclusive IgM-VH and IgG-VH sequences with identical mutations, suggesting evidence of local CSR (Table 1).

The $B$ cell repertoire in $m E L T$ is largely private. As a consequence of $\mathrm{V}(\mathrm{D}) \mathrm{J}$ recombination, addition of non-templated nucleotides, differential pairing of heavy and light chains, CSR, and SHM, the diversity of the B cell repertoire in humans and WT mice is extensive. However, in Th $\times 2 \mathrm{D} 2$ mice, the B cell repertoire is genetically restricted to a single rearranged germline Ig- $\mathrm{VH}$, with only SHM contributing to VH diversity. It would therefore be expected that the Ig-VH repertoire found in the various immunological compartments we investigated might be largely overlapping. Interestingly however, repertoire overlap with identical Ig-VH was surprisingly small, indicating that each of the sites generated their own private repertoires (Figure 3). When comparing mELT and SLO (i.e., lymph node [LN], and spleen), the percentage of identical CDR1CDR3 nucleotide sequences found in any two tissues was on average only $3.9 \%-7.0 \%$ (Figure $3 \mathrm{~B}$ ). None of the tissues had an entirely private repertoire, suggesting circulation of B cell clones between peripheral and 
A

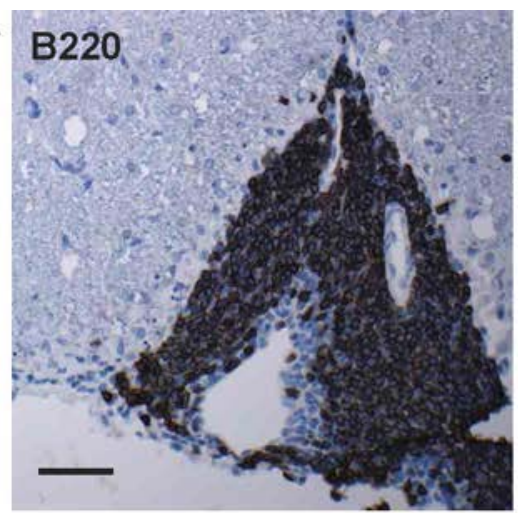

B

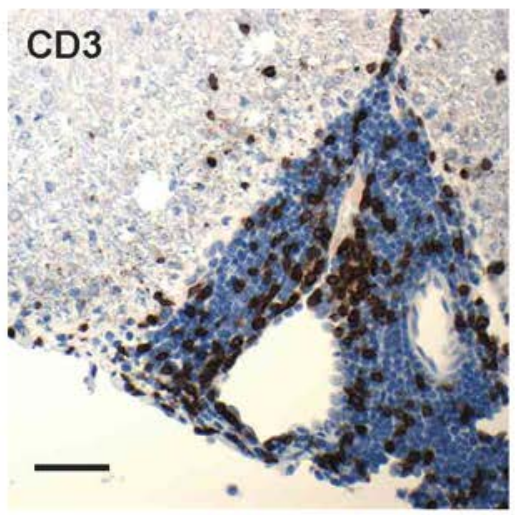

C

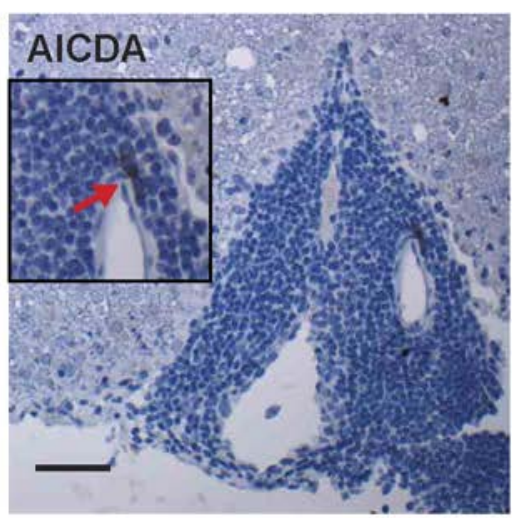

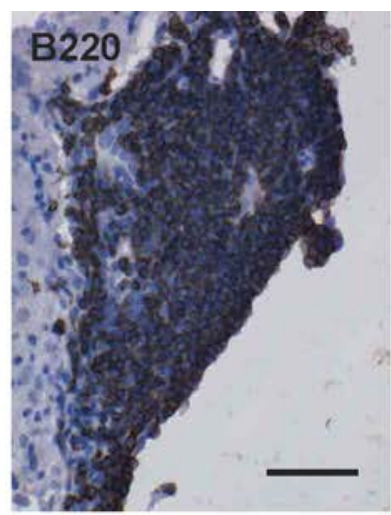
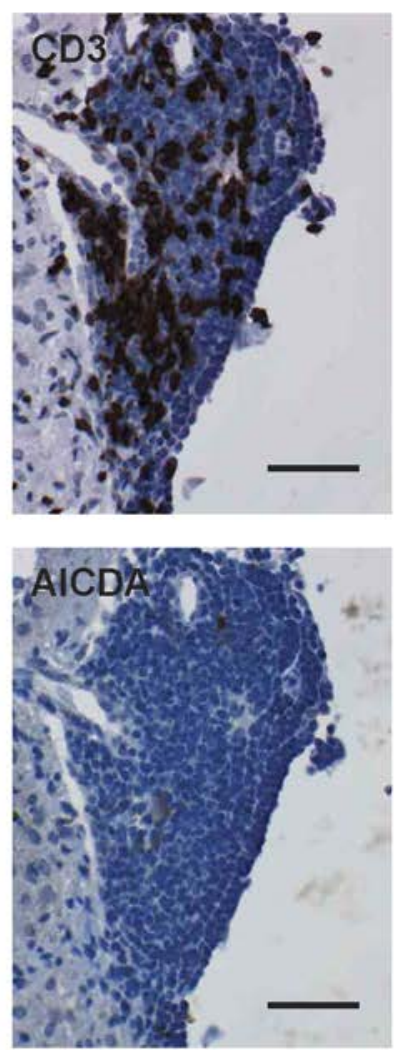

D

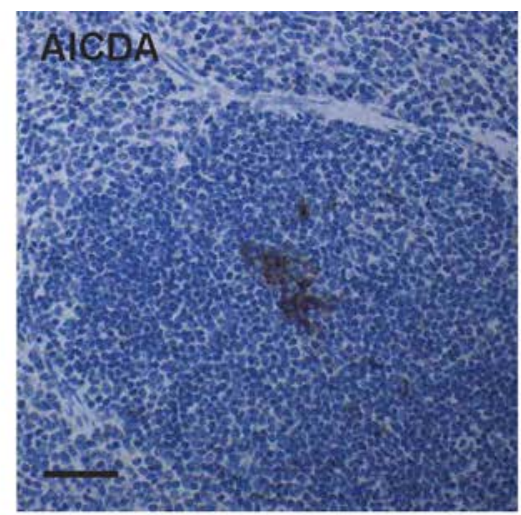

E

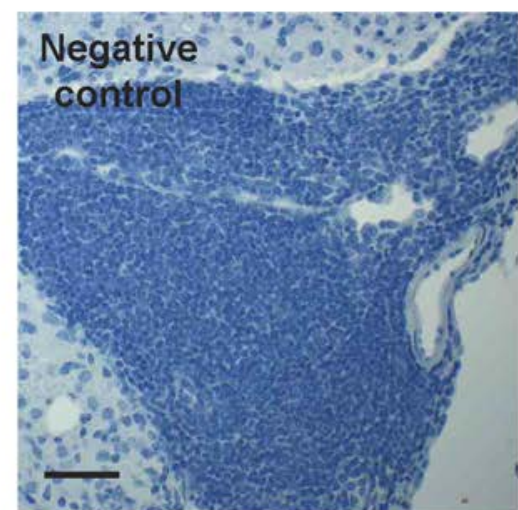

$\mathbf{F}$

S.c. relative

to kidney

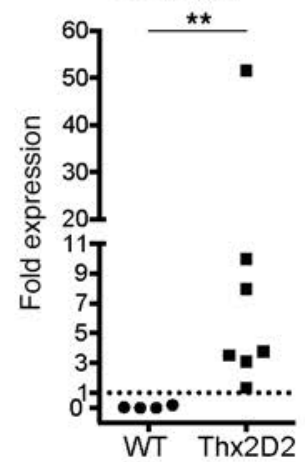

G Spleen relative

to kidney

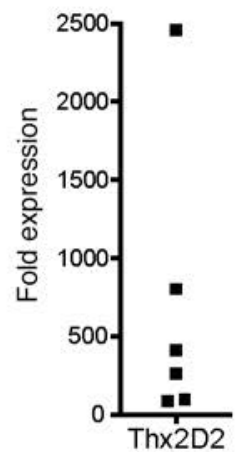

Figure 1. mELT contains $B$ and T cells and expresses AICDA. Presence of (A) B220+ (CD45R+) B cells and (B) $C D 3^{+} T$ cells, and (C) expression of AICDA in adjacent sections of spinal cord mELT from one Th×2D2 EAE mouse (representative of 3 mice). (D) AICDA expression in a splenic lymphoid follicle (positive control). (E) Negative staining control with the anti-rat Ig secondary Ab without a primary Ab. (F and $\mathbf{G}$ ) Relative expression of AICDA in the spinal cord (S.c.) relative to the kidney (F) or spleen relative to the kidney (C) from the same animal, using healthy WT $(n=4)$ or Th $\times 2 \mathrm{D} 2 \mathrm{EAE}$ mice $(n=7$ in $\mathbf{F}$ and $n=6$ in $\mathbf{G})$. qPCR (TaqMan); all values normalized to $\beta$-actin as reference gene. Each data point represents one mouse. Scale bars: $50 \mu \mathrm{m}$. ${ }^{* *} P=0.0061 ;$ Mann-Whitney $U$ test. AICDA, activation-induced cytidine deaminase; mELT, meningeal ectopic lymphoid tissue; EAE, experimental autoimmune encephalomyelitis.

CNS immune compartments, as was also seen in MS patients (24-26). Overall, these findings demonstrate that $\mathrm{mELT}$ participate in diversification of the B cell repertoire through SHM, qualifying them as true tertiary or ectopic lymphoid tissue.

mELT support antigen-driven somatic hypermutation. We examined whether the mutational patterns of B cell repertoires in $\mathrm{mELT}$ were consistent with SHM and antigen-driven affinity maturation. IgG-VH, representing class-switched post-GC B cells, displayed a high degree of SHM in EELT, comparable to SLO (spleen and LN; Figure 4, A-E). Conversely, IgM-VH displayed overall fewer mutations in all tissues studied, as expected for sequences derived from a mix of pre- and post-GC B cells expressing IgM (Figure 4F). Accordingly, significantly more IgM-VH sequences had a low rate of SHM (0-2 mutations) compared with 
A

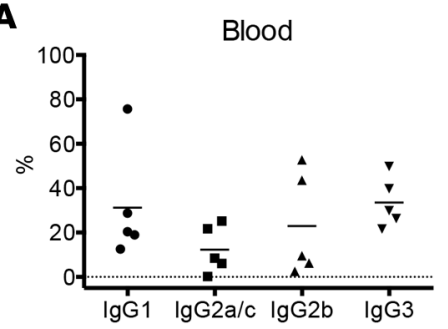

C

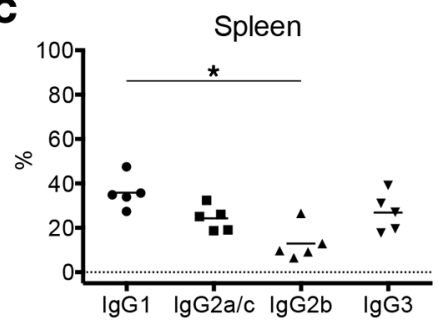

B

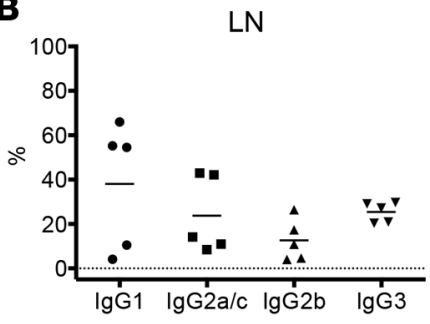

D

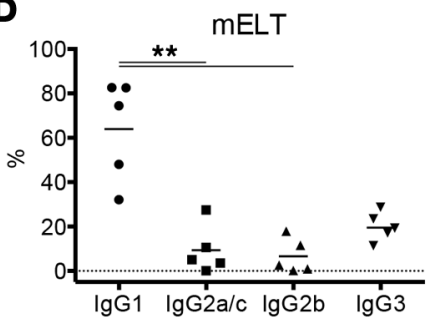

Figure 2. Class-switched B cells from mELT are preferentially of the IgG1 subclass. The frequency (\%) of IgG1, IgG2a/c, IgG2b, and IgG3 transcripts within all IgG sequences was determined in the blood (A), one LN (B), the spleen (C), and mELT (D) of 5 Th $\times 2 D 2$ EAE mice. Individual data points with mean are shown. ${ }^{*} P \leq 0.05$; ${ }^{* *} P \leq 0.01$; Friedman test with Dunn's multiple comparisons test. mELT, meningeal ectopic lymphoid tissue; LN, lymph node.

IgG-VH ( $P \leq 0.01$ for blood, LN, spleen, and mELT; 2-way ANOVA with Šidák's multiple comparisons test). Amino acid (aa) replacement mutations in Ig-VH resulting from SHM and consecutive antigen-driven selection of B cells are most commonly found in the CDRs, while they are underrepresented in the FRs (27). Kabat-Wu plots (28), graphing the frequency of replacement mutations along the IgG-VH ranging from CDR1 to CDR3, show that in mELT, and partially in other immune compartments, the majority of replacement mutations in class-switched B cells occur in the CDRs (Figure 5,

A-D). Comparison of normalized mutation rates in CDRs versus FRs showed that IgG-VH had accumulated significantly more mutations in CDRs in mELT and spleen but not in LN and blood (Figure 5E). This strongly suggested that antigen-driven SHM and selection contributed to B cell repertoires in mELT, similar to the spleen as a highly active site of B cell antigen training. Although IgG replacement mutations were clearly overrepresented in the CDRs in the spleen and in mELT, we found additional positions with high mutational activity within FRs (Figure 5, A-D) (29). Replacement mutations in IgM-expressing B cells, which may occur before or after GC reaction, were more randomly distributed along the entire IgM-VH in all tissues examined (Figure $5 \mathrm{~F}$ ), possibly representing trial-and-error BCR mutations, with less selection in pre-GC or non-class-switched B cells.

Replacement mutations in the Ig-VH CDR3 can readily alter binding affinity of an antibody/BCR. In Th $\times 2 \mathrm{D} 2$ mice, the germline Ig-VH CDR3 is pre-rearranged, i.e., somatic recombination of IGHV, IGHD, and IGHJ, and insertion of non-template nucleotides does not contribute to Ig-VH diversity. This enabled us to study the effects of SHM on CDR3 diversity. Extensive SHM resulting in aa replacements was observed in the CDR3, supporting antigen-driven affinity maturation (Figure 5, A-D, and Figure 6, A-C). We also investigated whether there was over-representation of certain mutations in the CDR3 in mELT compared with SLO (spleen and LN). At the level of individual mice, the frequency of specific aa was different between mELT and SLO, particularly at positions 106-108 and 110-113 of the CDR3. Examining all 5 mice together, we found 3 aa replacements to be significantly different between mELT and SLO (Figure 6): (a) CDR3 position 105 (D replaces germline A more frequently in mELT); (b) CDR3 position 109 (germline $\mathrm{T}$ is more conserved in $\mathrm{mELT}$ ); (c) CDR3 position 114 (L replaces germline $\mathrm{P}$ more frequently in $\mathrm{mELT}$ ). These findings suggest selection for certain CDR3 aa mutations in mELT, which may or may not result in altered affinity of antibodies and BCRs toward MOG, the principal autoantigen in $\mathrm{TH} \times 2 \mathrm{D} 2$ mice.

Ig lineage trees reveal SHM in mELT. In an effort to identify mELT-restricted Ig-VH lineages, we applied a computational approach specifically developed to calculate and visualize lineages of Ig variable region genes based on SHM (IgTree) (30). As expected, in each of the 5 individual mice, Ig-VH lineages were rather extensive, containing sequences that were either exclusively found in mELT (mELT-exclusive sequences/nodes; node $=$ all identical sequences represented together as one dot in the lineage tree) or in mELT and in one or more peripheral immune compartments (i.e., blood, spleen, LN; shared sequences/nodes). Importantly, however, in each mouse we also found at least two sublineages in which the majority or all Ig-VH were found exclusively in mELT (Figure 7, A and B, and Supplemental Figure 3). Many of these mELT-exclusive clusters (cluster = group of closely related nodes) were large, indicating extensive SHM. Interestingly, nodes unique to mELT were more likely to have a significantly larger number of cumulative mutations separating them from the germline (Figure 7C), again suggesting that mELT contribute to $\mathrm{BCR}$ repertoire evolution. In contrast, we also found clusters in which the majority of nodes were shared between mELT and one or more peripheral immune compartments. Those clusters were typically more 
Table 1. List of repertoire sequencing (RepSeq) reads

\begin{tabular}{|c|c|c|c|c|c|c|c|}
\hline Mouse no. & Compartment & $\begin{array}{l}\text { Redundant } \\
\text { reads[\# IgG] }\end{array}$ & $\begin{array}{l}\text { Nonredundant } \\
\text { reads ([\# IgG]) }\end{array}$ & $\begin{array}{c}\text { Redundant reads } \\
\text { [\# IgM] }\end{array}$ & $\begin{array}{l}\text { Nonredundant } \\
\text { reads[\# IgM] }\end{array}$ & $\begin{array}{c}\text { Identical IgM/G-VH } \\
\text { redundant [\# IgM/\# IgG] }\end{array}$ & $\begin{array}{l}\text { Identical IgM/G- } \\
\text { VH nonredundant }\end{array}$ \\
\hline A8 & Blood & 84553 & 3385 & - & - & $\mathrm{n} / \mathrm{a}$ & $\mathrm{n} / \mathrm{a}$ \\
\hline A8 & LN & 145832 & 15164 & - & - & $\mathrm{n} / \mathrm{a}$ & $\mathrm{n} / \mathrm{a}$ \\
\hline A8 & mELT 1 & 137333 & 13764 & - & - & $\mathrm{n} / \mathrm{a}$ & $\mathrm{n} / \mathrm{a}$ \\
\hline A8 & mELT 2 & 222717 & 10284 & - & - & $\mathrm{n} / \mathrm{a}$ & $\mathrm{n} / \mathrm{a}$ \\
\hline A12 & Blood & 115053 & 6872 & 309841 & 5459 & 63 / 976 & 55 \\
\hline A12 & LN & 108268 & 12175 & 156685 & 3439 & 83 / 767 & 71 \\
\hline A12 & Spleen & 146589 & 19476 & 243110 & 7980 & 224 / 2192 & 94 \\
\hline A12 & mELT 1 & 110979 & 15051 & 370845 & 9968 & 454 / 192 & 116 \\
\hline A12 & mELT 2 & 71914 & 10264 & 192441 & 3255 & $81 / 79$ & 69 \\
\hline A18 & mELT & 365978 & 7076 & - & - & $\mathrm{n} / \mathrm{a}$ & $\mathrm{n} / \mathrm{a}$ \\
\hline A20 & Blood & 305384 & 19665 & 434926 & 20200 & 329 / 740 & 244 \\
\hline A20 & LN & 257830 & 21167 & 388923 & 20135 & 620 / 1735 & 451 \\
\hline A20 & Spleen & 227718 & 43885 & 450058 & 22438 & 596 / 677 & 196 \\
\hline$A 20$ & mELT & 269845 & 8801 & - & - & $\mathrm{n} / \mathrm{a}$ & $\mathrm{n} / \mathrm{a}$ \\
\hline A22 & Blood & 132271 & 6042 & 147625 & 2683 & 16 / 4295 & 14 \\
\hline A22 & LN & 177538 & 4198 & 736235 & 9299 & 209 / 273 & 168 \\
\hline A22 & Spleen & 150108 & 22462 & 434726 & 7010 & 134 / 4960 & 69 \\
\hline A22 & mELT 1 & 81576 & 2781 & 546445 & 9207 & $0 / 0$ & 0 \\
\hline A22 & mELT 2 & 55221 & 2267 & 410276 & 6990 & $1 / 14$ & 1 \\
\hline A22 & mELT 1+2 & 136797 & 5042 & 956721 & 14691 & $1 / 14$ & 1 \\
\hline
\end{tabular}

Total number of nonredundant and redundant IgG and IgM sequencing reads obtained in different immune compartments from 5 Th $\times 2 \mathrm{D} 2$ mice with spontaneous EAE in the RepSeq analysis. All numbers are after bioinformatic processing (alignment, error correction, etc.). The last 2 columns list the number of identical redundant or nonredundant Ig-VH found both as IgM and IgG. They include only those sequences that were unique to the respective compartment and were not found in any other compartment in the same mouse. These sequences suggest CSR in the respective compartment. IgM-VH sequencing was not performed for mouse A8. For 2 additional mice (A18 and A20), we were unable to amplify sufficient IgM-VH transcripts from mELT for sequencing, probably because the majority of B cells in those mELT were class-switched. For mouse A8, A12, and A22, we were able to obtain tissue from more than one contiguous mELT (e.g., mELT 1, 2, 3). EAE, experimental autoimmune encephalomyelitis; VH, heavy chain variable region; CSR, class switch recombination; mELT, meningeal ectopic lymphoid tissue.

closely related to the germline (i.e., with fewer mutations separating them from the germline) (Figure 7C) and may represent B cells that underwent GC reaction in one lymphoid tissue and subsequently migrated to one or more other compartments.

For one mouse, we computed a joint IgG-IgM tree (Figure 7B). This tree confirmed that SHM occurs in non-class-switched (IgM) Ig sequences as well, but to a much lower degree, as the majority of IgM nodes are separated from the germline by only a few mutations.

As sequencing errors (substitution errors) may result in false nodes, they may potentially confound Ig lineage analyses. In order to virtually eliminate this possibility, we included only those nodes in Ig lineage trees that consisted of at least 3 identical reads. The upper band of the probability of the occurrence of sequencing errors causing one nucleotide substitution in 3 independent sequencing reads was calculated to be $\leq 5.08 \mathrm{E}-04$. However, the actual probability for these events is likely to be even lower, since the statistical model used (Poisson equation) does not account for the fact that sequencing errors would have to occur in the exact same position and to the same nucleotide in order to result in false nodes in Ig trees.

SHM occurring in mELT modifies Ig binding affinity. To address the question whether Ig utilizing VH that were mutated in mELT displayed altered binding affinity for the autoantigen MOG, we cloned a panel of mutated IgG-VH that were selected based on the following criteria: the IgG-VH sequences 
A
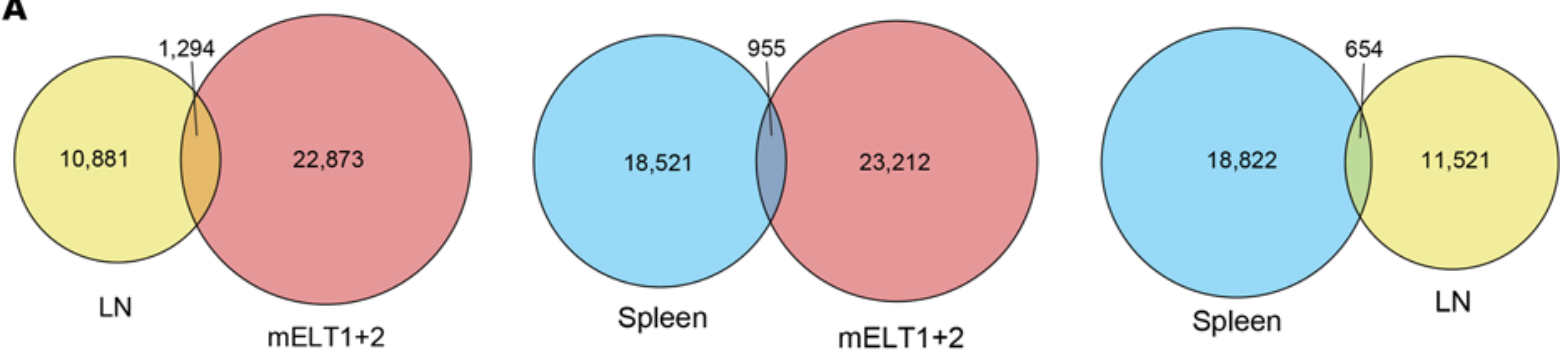

B
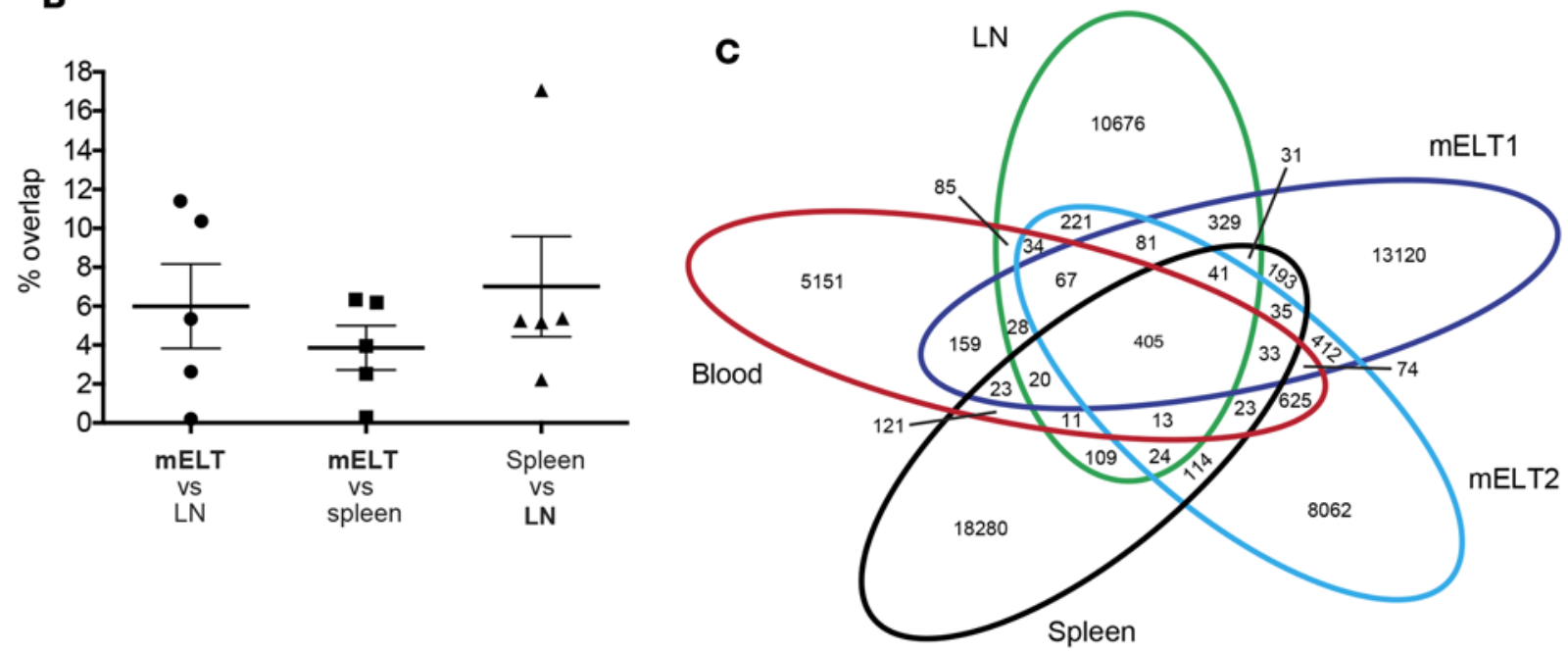

Figure 3. mELT and SLO each have their private Ig repertoires with relatively small overlap between each other. (A) Venn diagrams representing the number of unique IgG heavy chain sequences (comparing 2 compartments per diagram) that were found either exclusively in one compartment or in both compartments (overlap). The size of each circle is proportional to the count it represents. (B) Bar graphs representing the percentage (\%) of overlapping unique sequences between 2 compartments of all unique sequences in the designated reference compartment (in bold in the $x$-axis label) are shown. Mean of $5 \mathrm{Th} \times 2 \mathrm{D} 2 \mathrm{EAE}$ mice is shown (error bars, SEM). Differences between groups were not significant (Friedman test with Dunn's multiple comparisons test). (C) Venn diagram representing the number of unique lgG heavy chain sequences in various immune compartments (blood, LN, spleen, and 2 spatially independent spinal cord mELT) and their overlap in all possible combinations. (A and C) Data from one Th $\times 2 D 2$ EAE mouse (representative of 5) are shown. (A-C) The entire region starting with the first nucleotide of the CDR1 and extending to the last nucleotide of the CDR3 is included. mELT, meningeal ectopic lymphoid tissue; SLO, secondary lymphoid organ; EAE, experimental autoimmune encephalomyelitis; LN, lymph node; CDR, complementarity determining region.

were detected exclusively in mELT as a member of an IgG-VH sublineage reflecting local SHM; within a sublineage, the most frequently encountered IgG-VH sequence (i.e., highest number of identical reads) was selected. The location of the sequences within Ig lineage trees is shown in Figure 7A and Supplemental Figure 3B. IgG-VH that were representative of SHM occurring in mELT of the respective individual mouse were chosen (compare Figure 5 for reference). We selected a total of $10 \mathrm{IgG}-\mathrm{VH}$ from 2 mice (A12 and A20) displaying 2-8 aa replacements when compared with the germline sequence (Figure 8A). Each IgG-VH was cloned into an expression plasmid and coexpressed with the kappa $\mathrm{L}$ chain (Igא-VL) from the 8.18-c5 hybridoma to yield a complete IgG1 rmAb. Nine of the $10 \mathrm{rmAb}$ could be successfully expressed in sufficient amounts for binding studies via biolayer interferometry. The original germline Ig-VH was also subcloned into the same expression system, resulting in rm8.18-c5. In 33 repeated measurements, the MOG binding affinity of rm8.18-c5 was highly consistent (mean $K_{D}=2.12$ $\pm 0.07 \mathrm{nM}, \mathrm{SEM}$ ). Thus, binding studies for each $\mathrm{rmAb}$ included $\mathrm{rm} 8.18$-c5 as an internal reference,

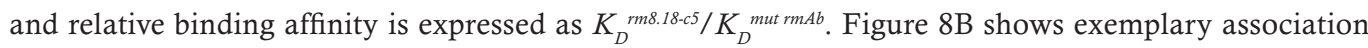
and dissociation sensorgrams for $\mathrm{rmAb} \mathrm{A} 12-\mathrm{H} 2$ and its reference $\mathrm{rmAb}, \mathrm{rm} 8.18$-c5. Two of the $9 \mathrm{rmAb}$ showed no detectable binding to MOG (A12-H6 and A20-H1; Figure 8C). Those rmAb had mutations in both their CDR2 and CDR3 that were not present in any of the other rmAbs (Figure 8A). Two other $\mathrm{rmAb}$ demonstrated weak MOG binding (A12-H9 and A20-H2) and $3 \mathrm{rmAb}$ were strong MOG binders (A12-H4/-H8/-H10), with an affinity comparable to rm8.18-c5 (Figure 8C). Interestingly, 2 

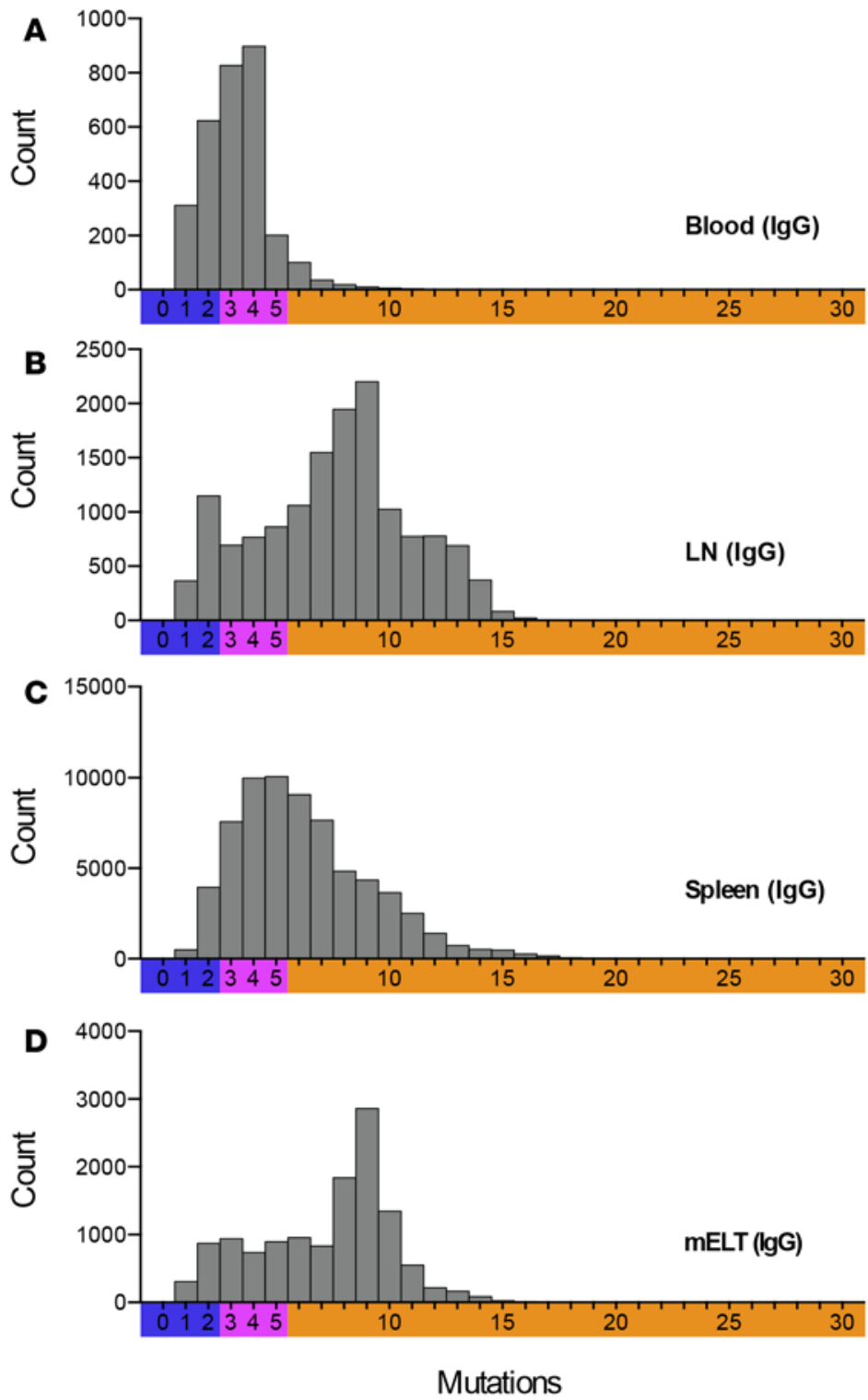

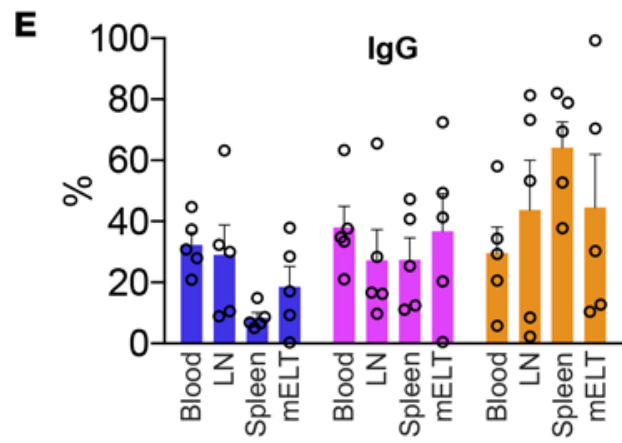

$\mathbf{F}$

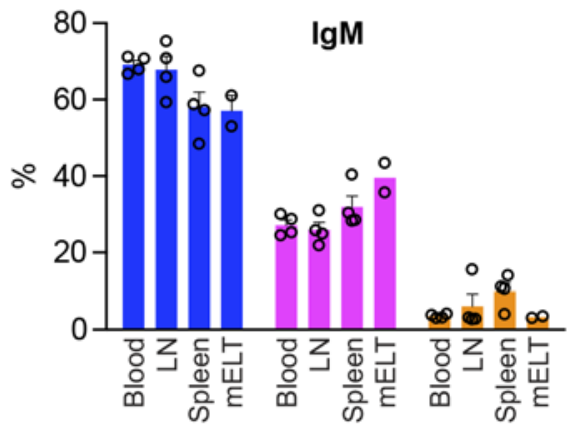

Figure 4. IgG transcripts from mELT exhibit a high frequency of SHM comparable to SLOs, while IgM transcripts exhibit a minor frequency of SHM in all compartments. Count of nonredundant IgG (A-E) and IgM (F) heavy chain sequences according to their number of mutations in the VDJ region, starting with the first nucleotide of the CDR1 and extending to the last nucleotide of the CDR3. Data from the blood (A), an inguinal LN (B), the spleen (C), and mELT (D) from one representative Th $\times 2$ D2 EAE mouse are shown $(n=5$ mice). (E and $\mathbf{F}$ ) Frequency of SHM is grouped into 3 categories: low ( $0-2$ mutations), intermediate (3-5 mutations), and high ( $\geq 6$ mutations) SHM. Mean of 5 (E) and 4 (F) mice is shown for each of the 4 compartments, except for lgM in $\mathrm{MELT}(\mathbf{F})$, where data points for only 2 mice were available (error bar, SEM). Differences between compartments within each category were not significant in $\mathbf{E}$ or $\mathbf{F}$; 2-way ANOVA with Dunnett's multiple comparisons test. However, significantly more IgM-VH sequences (F) had a low rate of SHM (0-2 mutations) compared with IgG-VH (E); $P \leq 0.01$ for blood, LN, spleen, and mELT; 2-way ANOVA with Šidák's multiple comparisons test. mELT, meningeal ectopic lymphoid tissue; SHM, somatic hypermutation; SLO, secondary lymphoid organ; CDR, complementarity determining region; LN, lymph node; EAE, experimental autoimmune encephalomyelitis; $\mathrm{VH}$, heavy chain variable region.

rmAbs exhibited higher MOG binding affinity than rm8.18-c5 (A12-H2, $1.73 \pm 0.12$-fold relative binding affinity; A20-H8, $1.19 \pm 0.07-$ fold relative binding affinity; Figure 8C). A12-H2, the rmAb with the highest MOG affinity, has a CDR3 replacement mutation (N108 $\rightarrow$ D108), an aa substitution commonly observed among mELT Ig-VH (Figure 6, A-C), in addition to another mutation in the CDR3 $(\mathrm{A} 105 \rightarrow \mathrm{V} 105)$ and one in the FR3 $(\mathrm{K} 71 \rightarrow \mathrm{N} 71)$. A20-H8 also exhibited increased MOG affinity, with only a single replacement in the FR3 $(\mathrm{E} 81 \rightarrow \mathrm{V} 81)$, suggesting that single aa changes outside the antigen-binding site can alter binding affinity. 
A
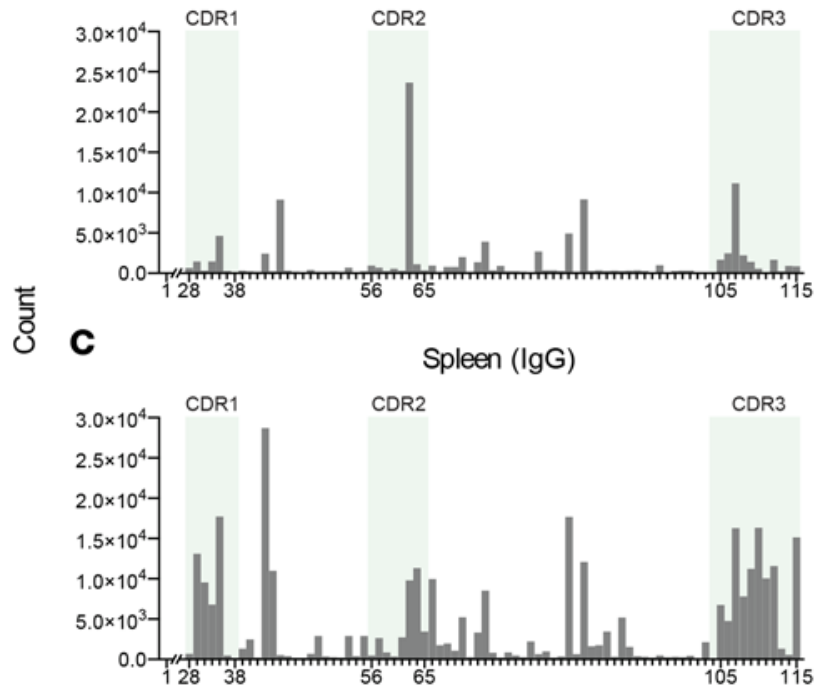

B

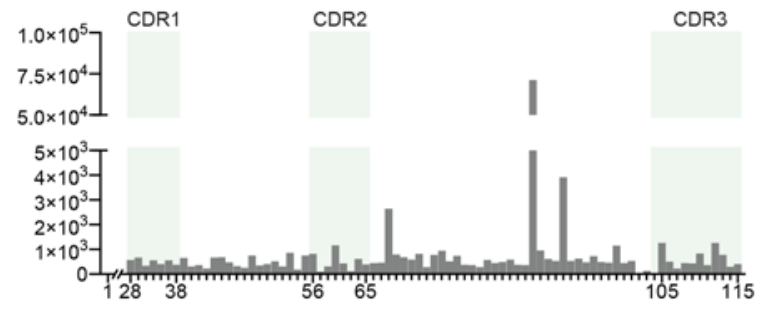

D

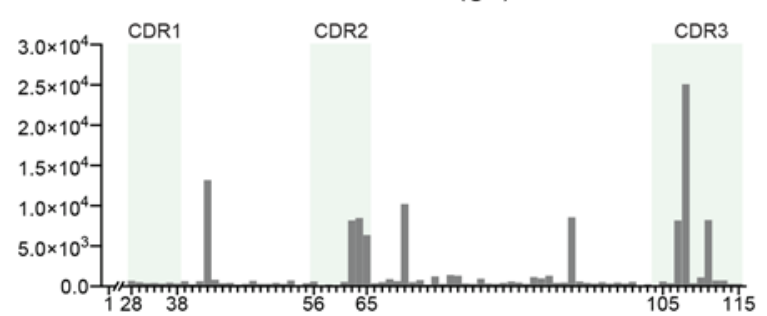

AA position
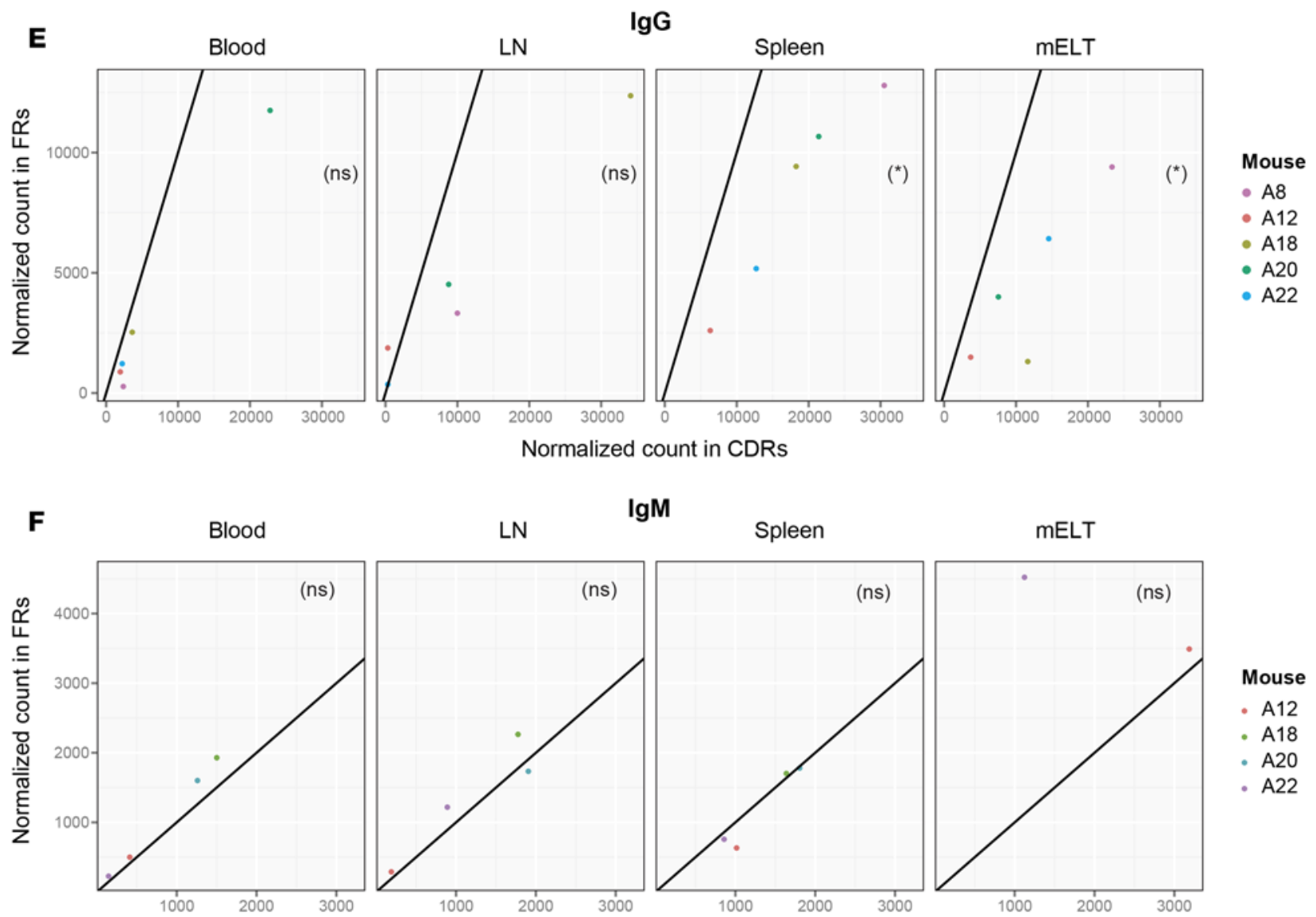

LN

IgM

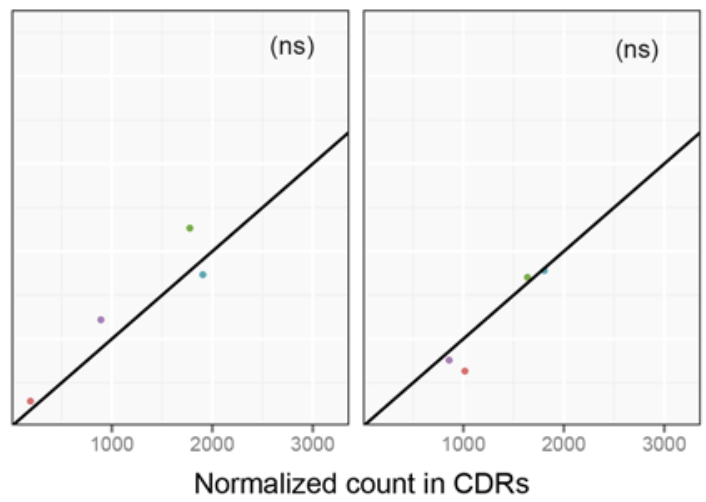

Spleen mELT

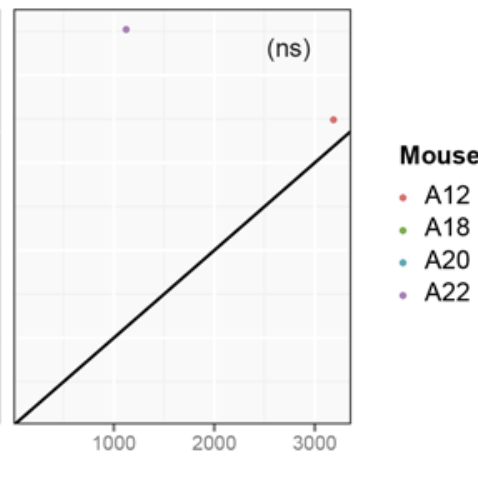

Figure 5. SHM in IgG but not IgM transcripts from mELT occurs in an antigen-driven fashion and exhibits a profile distinct from that in other, peripheral immune compartments. (A-D) The frequency of aa mutations in an 81-aa portion of the VDJ region, starting with the first aa of the CDR1 (IMGT position 28) and extending to the last aa of the CDR3 (IMCT position 115), is plotted. Only lgG sequencing reads are included. Data from the blood (A), an inguinal LN (B), the spleen (C), and mELT (D) from one representative Th×2D2 EAE mouse are shown ( $n=5$ mice). CDR1, CDR2, and CDR3 according to IMCT are highlighted in green. (E and F) Cumulative mutation counts in the CDRs (CDR1, CDR2, and CDR3 together) and the framework regions (FR2 and FR3 together) were normalized separately for their respective length and plotted against each other. Data from the blood, LN, spleen and mELT are shown from (E) 5 Th $\times 2 D 2$ EAE mice or (F) 4 Th×2D2 EAE mice (for mELT, only 2 data points were available), respectively. Data points located to the right of the black line indicate overrepresen- 
tation of SHM in the CDRs compared with the FRs, suggesting an antigen-driven process. ns, not significant; ${ }^{*} P \leq 0.05$; paired 2 -tailed $t$ test adjusted for multiple comparison using the Benjamini-Hochberg method. SHM, somatic hypermutation; mELT, meningeal ectopic lymphoid tissue; CDR, complementarity determining region; LN, Iymph node; EAE, experimental autoimmune encephalomyelitis; IMGT, International ImMunoGeneTics; FR, framework region.

\section{Discussion}

B cell-rich follicle-like structures have been described in EAE (17-21) and in SPMS (12-15), but it is unclear whether mELT support GC function. Here, the rearranged Ig-VH expressed by all B cells in a mouse model that develops spontaneous chronic EAE was used as a template to examine SHM and CSR in mELT and SLO from the same animals. We demonstrated expression of AICDA, SHM, and CSR, functional aspects of GC reaction in $\mathrm{mELT}$, indicating that $\mathrm{B}$ cells within this CNS immune compartment actively participate in autoimmunity in situ.

Evidence of AICDA expression has been reported in ELT in patients with various autoimmune diseases and in animal models (e.g., rheumatoid arthritis, ref. 3; murine lupus, ref. 8; Sjögren's disease, ref. 4; and SPMS, ref. 5), and was also present in mELT studied here. In Th×2D2 mice with clinical EAE, we demonstrated by next-generation RepSeq analysis of mELT B cell repertoires that AICDA-dependent GC functions, specifically SHM and CSR, occurred in mELT. To our knowledge, this is the first study to apply this technology to ELT in general, and to mELT in CNS autoimmune disease in particular. Accumulation of replacement mutations in Ig-VH CDRs, structural features directly responsible for the binding of BCR or antibodies to their cognate antigen, suggested that GC activity in mELT was antigen driven. Several studies have investigated clonally related B cells present in ELT in Lyme arthritis (7), murine (8) and human (9) lupus, rheumatoid arthritis (10), and breast cancer (11). In addition, B cell clones found in meningeal B cell aggregates of SPMS patients were related to those in parenchymal MS lesions (31). However, due to technical limitations those studies did not investigate whether SHM had actually occurred in the respective target organ, or if the presence of clonally related B cells was due to directed migration and/or selective accumulation. Through deep sequencing technology, we were able to comprehensively analyze B cell repertoires in mELT and SLO. We detected numerous mutated Ig-VH within mELT that were not identified in SLO. The presence of unique B cells in mELT reflected in situ GC activity and could not have been merely due to CNS migration and expansion of B cells that had undergone diversification in the periphery. Our results therefore support the notion that these tertiary immune tissues in the meninges represent partially autonomous sites of immune activity, rather than immigrated B cell populations. A recent study demonstrated that clonally related B cells are present in the brain (including MS lesions) and brain-draining cervical LN in MS patients, with founding members of these clonal families more frequently present in cervical LN (25). This confirmed earlier findings suggesting that B cells exchange between the CNS and the periphery (26). Since mELT were not specifically subject of the study by Stern et al. (25) and CNS-draining LN were not subject of ours, future work should investigate how the B cell repertoires of mELT, the CNS parenchyma (lesions), and CNS-draining LN relate to each other.

We demonstrated that ectopic GC reaction in the meninges could have significant consequences for the binding properties of Ig genes expressed in mELT. In this regard, we generated a number of rmAb by pairing the 8.18-c5 hybridoma Igא-VL with IgG-VH found exclusively in mELT, to compare rmAb MOG binding capabilities with the parent 8.18-c5 MOG-specific antibody. We observed that IgG-VH replacement mutations introduced in mELT increased, decreased, or even eliminated MOG-binding capacity, demonstrating that SHM in mELT may directly modulate B cell immunity. By applying RepSeq rather than single-cell BCR analysis for our studies, we gave priority to comprehensive information on tissue-specific Ig-VH repertoires that only next-generation sequencing technologies can provide. Accordingly, which Igא-VL or Ig $\lambda$-VL naturally paired with mELT Ig-VH is unknown. Given that previous studies have shown that the Ig light chain variable region has only limited influence on MOG binding when paired with the knockin 8.18-c5 $\operatorname{IgH}(32)$, we are confident that our approach faithfully replicated the potential effects of Ig-VH SHM in mELT on MOG binding of locally expressed BCR and Ig. B cell autoimmunity against MOG, an established CNS autoantigen, is a hallmark feature of $\mathrm{Th} \times 2 \mathrm{D} 2$ mice; $30 \%-50 \%$ of $\mathrm{B}$ cells in $\mathrm{Th} \times 2 \mathrm{D} 2$ mice are MOG reactive despite diverse usage of light chain genes $(20,33,34)$. In spontaneous $\mathrm{EAE}$ in $\mathrm{TH} \times 2 \mathrm{D} 2$ mice, recognition, processing, and presentation of the encephalitogenic MOG protein requires B cells as principal antigen-presenting cells (APCs) (20). Accordingly, mELT may be capable of controlling not only the binding affinity of soluble Ig and associated effector functions, but also the efficiency of antigen capture by the BCR and thus the antigen-presenting function of B cells. Increasing the BCR affinity may enhance antigen capture and presentation, and therefore sustain or even enhance local inflammation and CNS tissue destruction. 
A

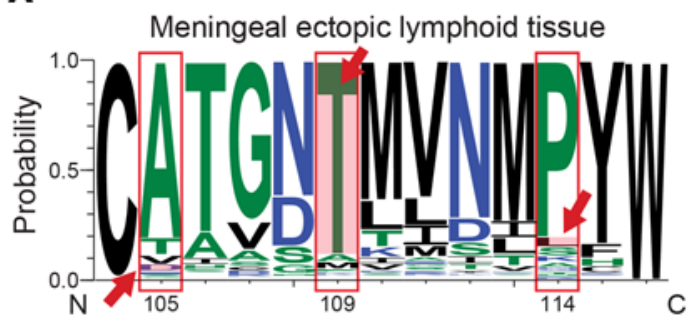

B

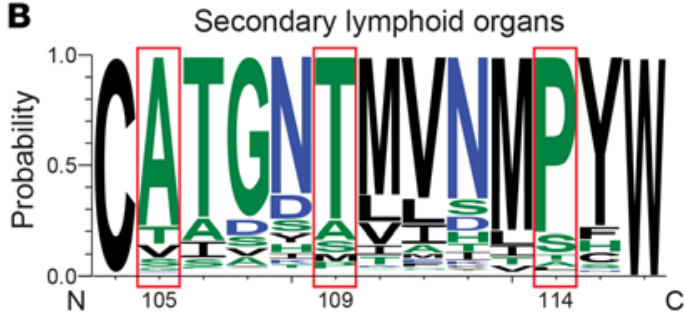

C

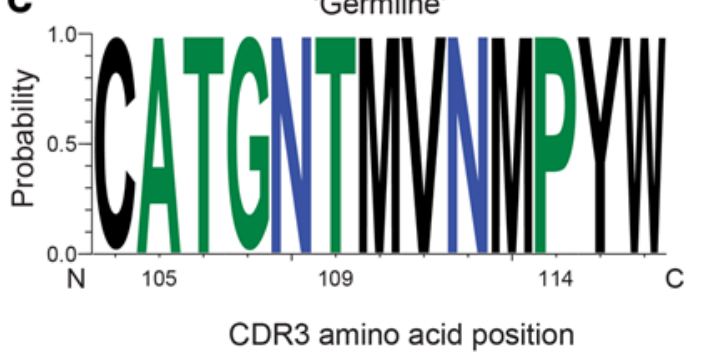

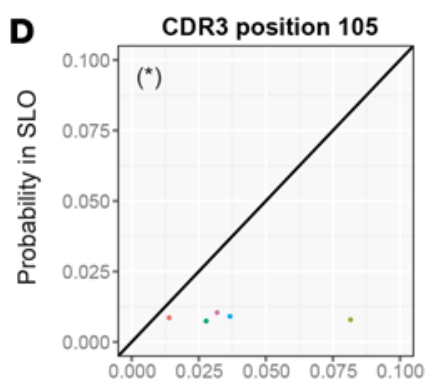

$\mathbf{E}$
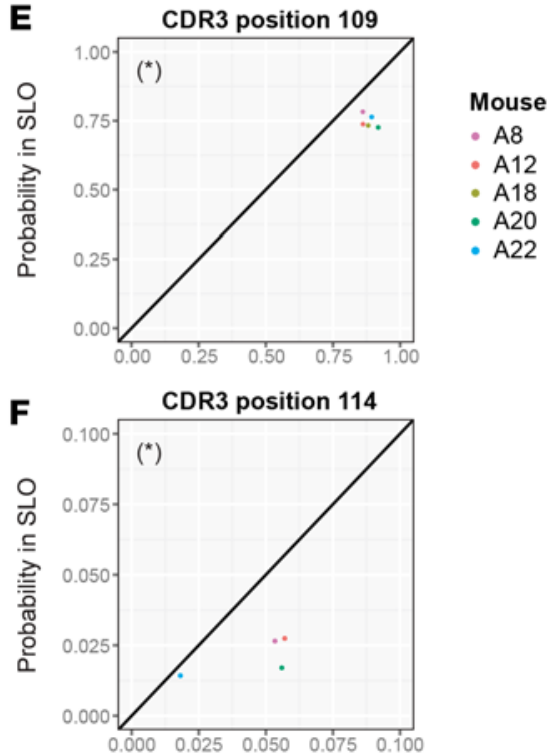

Probability in mELT

Figure 6. CDR3s from IgG transcripts present in mELT feature a pattern of a mutations distinct from that in SLOs. (A-C) Logos depict a graphical representation of the probability of any aa to occur in the 13 aa positions of the IgG-VH CDR3 region, reflecting SHM. The mean probability in (A) mELT and (B) SLO is shown. (C) The germline CDR3 sequence from the 8.18-c5 knockin Ig-VH served as the reference sequence. Amino acid positions are according to IMCT, and letter colors are according to hydrophobicity (hydrophilic, blue; neutral, green; hydrophobic, black). N, N-terminus; C, C-terminus. Those aa positions with significant differences ( $P \leq 0.05$; permutation test based on 9999 Monte-Carlo resamplings) between mELT and SLO are marked with red boxes in $\mathbf{A}$ and $\mathbf{B}$, and the respective aa are highlighted with a red background and arrow in A. (D-F) The probabilities for specific aa to be present in specific positions of the CDR3 in mELT (X-axis) or SLO (y axis) are plotted against each other. (D) CDR3 position 105: replacement of the germline aa alanine with aspartate; (E) CDR3 position 109: conservation of the germline aa threonine; (F) CDR3 position 114: replacement of the germline aa proline with leucine. Data points located to the right of the black line indicate overrepresentation of the specific aa in mELT compared with SLO at this specific position of the CDR3. For mouse A18, there are no data points in $\mathbf{F}$ because no leucine was detectable in SLOs, and this mouse had to be excluded from this part of the analysis. ${ }^{*} P \leq 0.05$; permutation test based on 9999 Monte-Carlo resamplings. (A-F) All IgG sequences from 5 Th $\times 2 D 2$ EAE mice are included, unless stated otherwise above. SLO = spleen and one inguinal LN combined. CDR, complementarity determining region; mELT, meningeal ectopic lymphoid tissue; SLO, secondary lymphoid organ; VH, heavy chain variable region; SHM, somatic hypermutation; IMGT, International ImMunoGeneTics; EAE, experimental autoimmune encephalomyelitis; LN, lymph node.

Reducing the BCR repertoire complexity to a single rearranged Ig-VH germline sequence, such as in the disease model used here, greatly simplified analyses of immune repertoires and local GC activity by RepSeq. This point is important, considering that in humans or WT animals, Ig-VH mutation analyses are restricted to the IGHV germline-derived sequence portion, while the randomness involved in the recombination events resulting in the CDR3 precludes SHM analysis of the region that is most relevant to antigen-binding. Due to the genetically fixed rearranged Ig-VH CDR3, the Th $\times 2 \mathrm{D} 2$ model of spontaneous EAE may currently be the only experimental model permitting reliable lineage analyses that includes the CDR3. While next-generation sequencing has greatly advanced the field of immune repertoire analysis, sequencing errors continue to be a concern in Ig RepSeq. Collateral experiments determining the sequenc- 


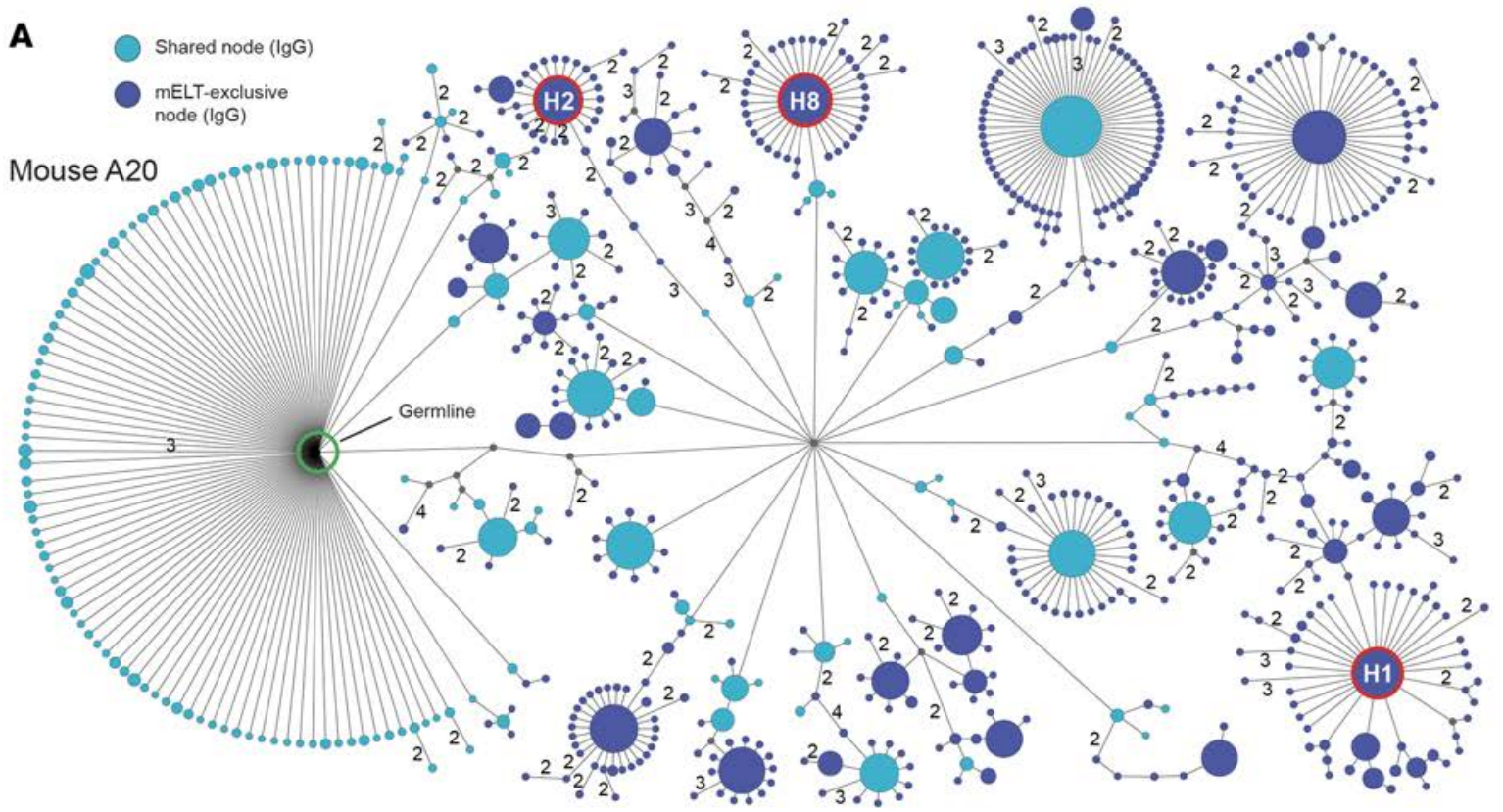

B

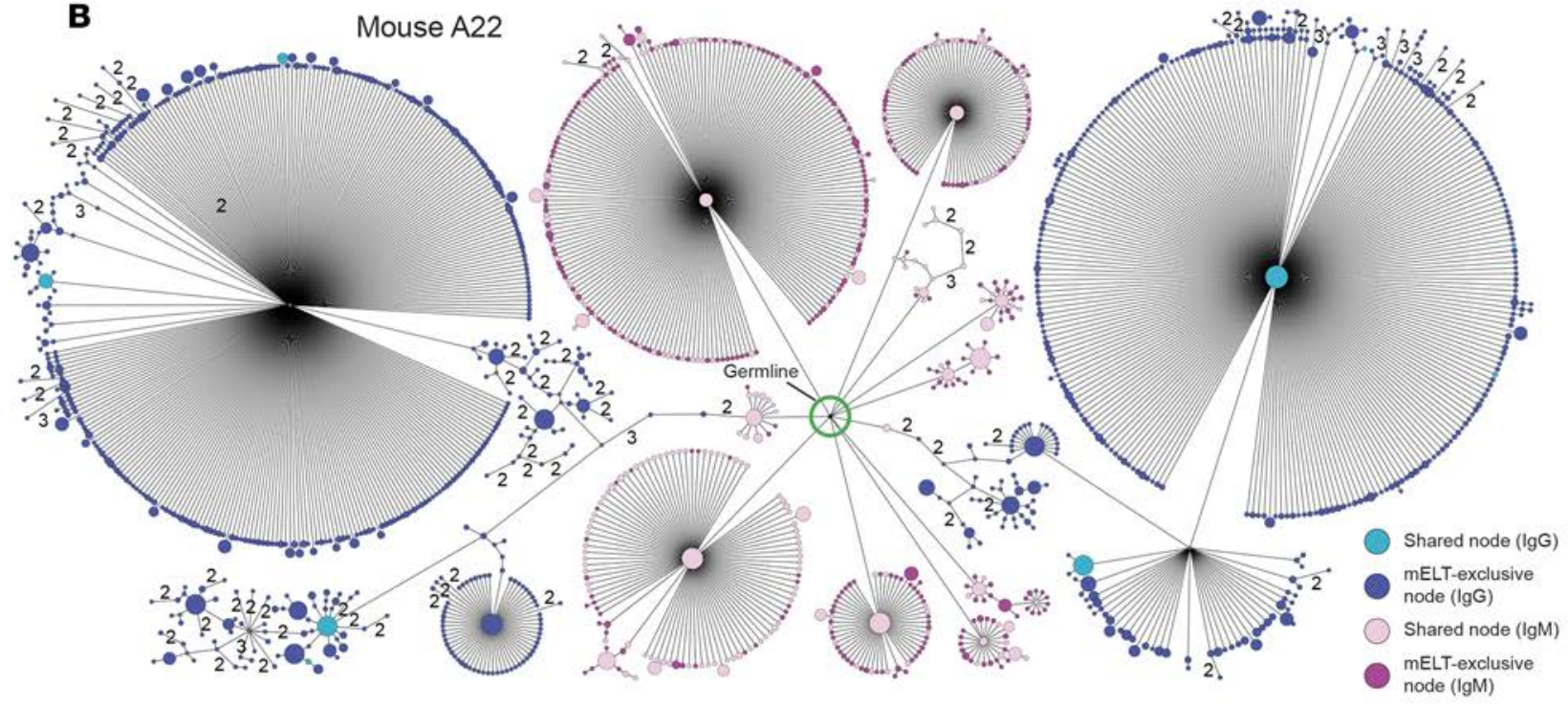

C

Number of mutations

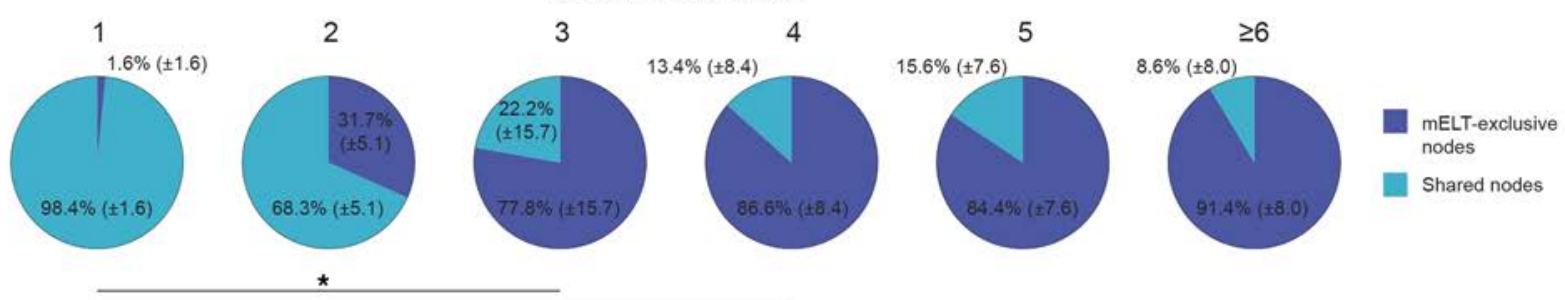

Figure 7. Ig lineage trees evidence intrinsic affinity maturation in mELT. (A) IgG lineage tree from mELT from Th $\times 2 D 2 E A E$ mouse A20. (B) IgG and IgM lineage tree from mELT from Th×2D2 EAE mouse A22. (A and B) Each node represents all sequencing reads with an identical CDR1-CDR3 region. To reduce the risk that rare sequencing errors result in false nodes, only those nodes with $\geq 3$ identical sequences are shown. Dark blue (IgG) or red (IgM) nodes are sequences found exclusively in mELT. Turquoise (IgG) or pink (IgM) nodes are sequences found in mELT and one or more peripheral compartments (blood, spleen, LN). Shared nodes; mELT, peripheral compartments. Black nodes highlighted with a green circle represent the germline sequence (knockin lg-VH). Gray nodes represent sequences that were not found in the sequencing data but were computed to complete the tree (internal nodes). The size of each node correlates with the number of identical sequencing reads. Two nodes connected by a line differ from each other by one particular nucleotide mutation (if not indicated differently by a number) in a specific position of the entire CDR1-CDR3 region. In $\mathbf{A}$, sequences that were selected 
for subsequent cloning and expression are labeled (e.g., "H1") and highlighted with a red circle. (C) Nodes in the lgG lineage trees of $5 \mathrm{Th} \times 2 \mathrm{D} 2 \mathrm{EAE}$ mice were categorized into groups according to the number of mutations relative to the germline sequence $(1,2,3,4,5$, $\geq 6$ mutations). For each category, the mean percentage \pm SEM of nodes that were found either exclusively in mELT or that were found in mELT and one or more peripheral compartments (blood, spleen, LN) is given. Shared nodes; mELT, peripheral compartments. ${ }^{*} P \leq 0.05$; ${ }^{*} P \leq 0.01$; Kruskal-Wallis test with Dunn's multiple comparisons test. mELT, meningeal ectopic lymphoid tissue; EAE, experimental autoimmune encephalomyelitis; CDR, complementarity determining region; $\mathrm{LN}$, lymph node; $\mathrm{VH}$, heavy chain variable region.

ing error rate and probability were used to establish a stringent bioinformatics analysis pipeline, thereby minimizing potential confounding effects of sequencing errors in our analyses of SHM and B cell lineages.

In summary, our data provide functional evidence for ectopic GC reaction in ELT. In EAE, and possibly MS, mELT are sites of local immune activity resulting in production of autoantibodies with modified affinity for myelin self-antigens, which may directly influence local immune activity. Antigen-specific BCR engagement has a profound influence on B cell APC function $(20,35,36)$. Thus, it is conceivable that an increase or decrease in BCR affinity for myelin protein may also alter efficiency of APC function and secretion of proinflammatory cytokines that promote CNS tissue destruction. Our observations that mELT participate in the CNS immune response may be relevant to MS therapy. Despite the advent of highly effective MS treatments, including anti-CD20 B cell-depleting monoclonal antibodies $(37,38)$, experimental and human evidence suggests that B cells within GCs in secondary and tertiary lymphoid organs may be resistant to anti-CD20-mediated depletion $(39,40)$. In fact, ELT in therapy-inaccessible B cell niches may pose a significant conceptual roadblock in the development of monoclonal antibody therapies against SPMS and other chronic inflammatory diseases associated with tertiary lymphoid tissues. Thus, one may ask whether either preventing the formation of ELT or selectively targeting gene products associated with GC reaction could provide an effective approach to ameliorate progressive CNS tissue damage in MS.

\section{Methods}

Mice and spontaneous EAE. $\mathrm{MOG}_{35-55}$-reactive TCR transgenic (2D2, TCR ${ }^{\mathrm{MOG}}$ ) mice (41) were a gift of V.K. Kuchroo (Harvard University, Cambridge, Massachusetts, USA). MOG-BCR knockin (IgH ${ }^{\text {MOG-ki }}$, also referred to as Th) mice (33) were a gift of $\mathrm{H}$. Wekerle (Max Planck Institute of Neurobiology, Martinsried, Germany). Both strains were on the C57BL/6 background. Th mice were crossed with 2D2 mice to generate $\mathrm{Th} \times 2 \mathrm{D} 2$ mice $(21,22)$, which develop spontaneous EAE with mELT. Important for our studies, $\mathrm{Th} \times 2 \mathrm{D} 2$ mice develop normal immune organs and lymphoid follicular structures despite the presence of a single rearranged knockin germline Ig-VH. C57BL/6 WT control mice were bred in-house. Individual animals were observed daily by a rater who was not blinded to the experimental protocol for signs of EAE, and clinical scores were assessed with a 0 - to 5-point scoring system, as follows: 0 , no clinical disease; 1, loss of tail tone only; 2, mild monoparesis or paraparesis; 3, severe paraparesis; 4, paraplegia and/or quadriparesis; and 5, moribundity or death. Moribund mice were given disease severity scores of 5 and euthanized. Animals were housed in a specific pathogen-free barrier facility at the Sandler Neurosciences Center of UCSF.

Tissue preparation for RepSeq studies. Five female $\mathrm{Th} \times 2 \mathrm{D} 2$ mice with clinical EAE (EAE score $>3$ ), which were randomly chosen from our colony of Th $\times 2 \mathrm{D} 2$ mice, were sacrificed 15-47 days after disease onset (Supplemental Table 1 lists their clinical characteristics). Blood (0.3-0.5 ml) was collected via cardiac puncture. After erythrocyte lysis, leukocytes were washed. After thorough perfusion with PBS, the spleen, one inguinal LN, and the spinal cord were gently dissected, preserving the meninges. The spinal cord was snap-frozen in Tissue-Tek O.C.T. (Sakura) in an isopentane/dry ice bath and transferred to $-80^{\circ} \mathrm{C}$ until further analysis. Cells were isolated from half of the spleen and the inguinal LN by crushing, passing through $70-\mu \mathrm{m}$ cell strainers (BD), and washing. From these cells, RNA was isolated immediately.

LCM. Transverse sections of the spinal cord at various levels were cut using a cryostat-microtome (thickness $20 \mu \mathrm{m}$ ). Precautions were taken to prevent cross-contamination or exposure to RNase. Samples were kept on dry ice before and after cutting. Immediately after cutting, slices were stained using the HistoGene LCM Frozen Section Staining Kit (Arcturus, Applied Biosystems) following the manufacturer's instructions and then underwent LCM. mELT, which are areas of cell-rich infiltrates in the meninges of the spinal cord, were visually identified, marked, cut, and captured (Supplemental Figure 1) in all 5 mice using Arcturus Veritas Microdissection Instrument 704 with Arcturus CapSure Macro LCM Caps and Veritas 
A

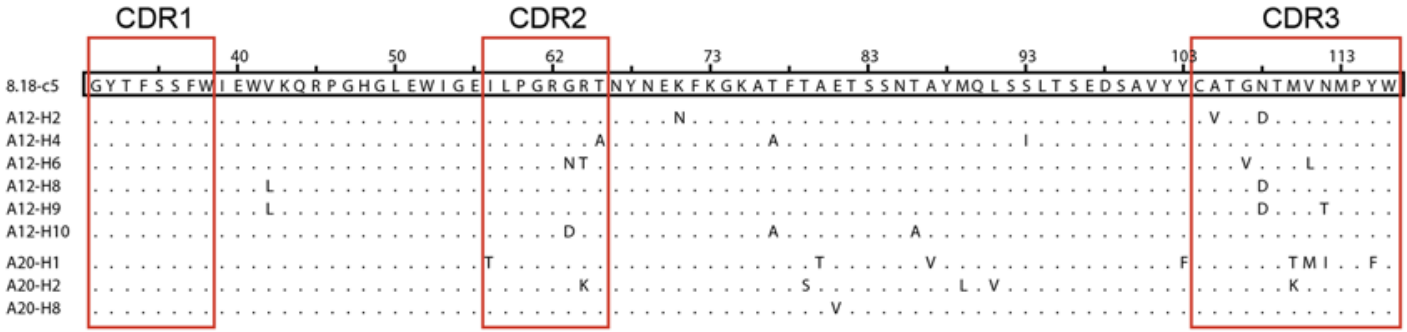

\section{B}

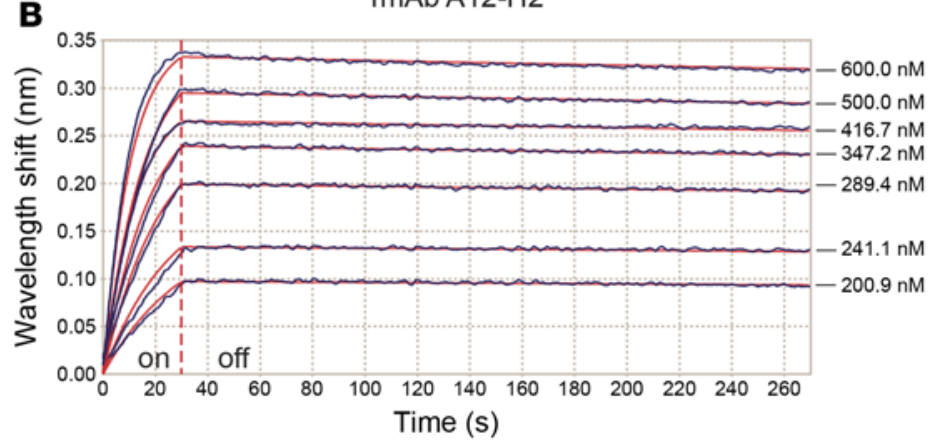

rm8.18-c5

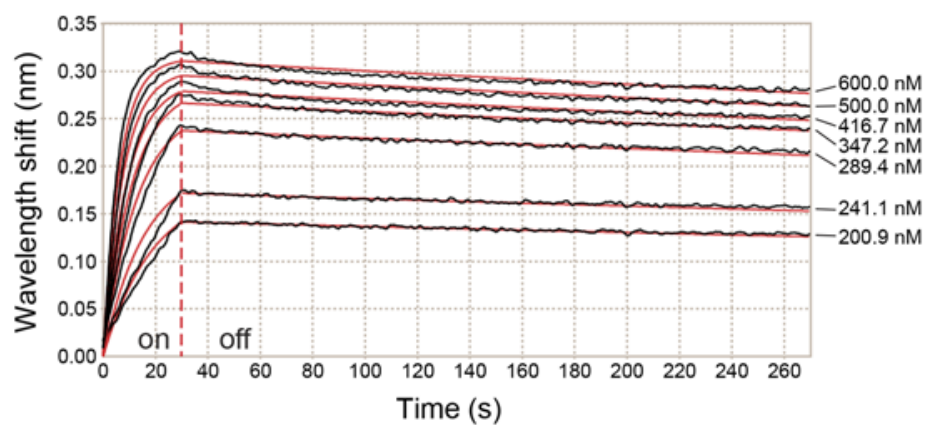

C
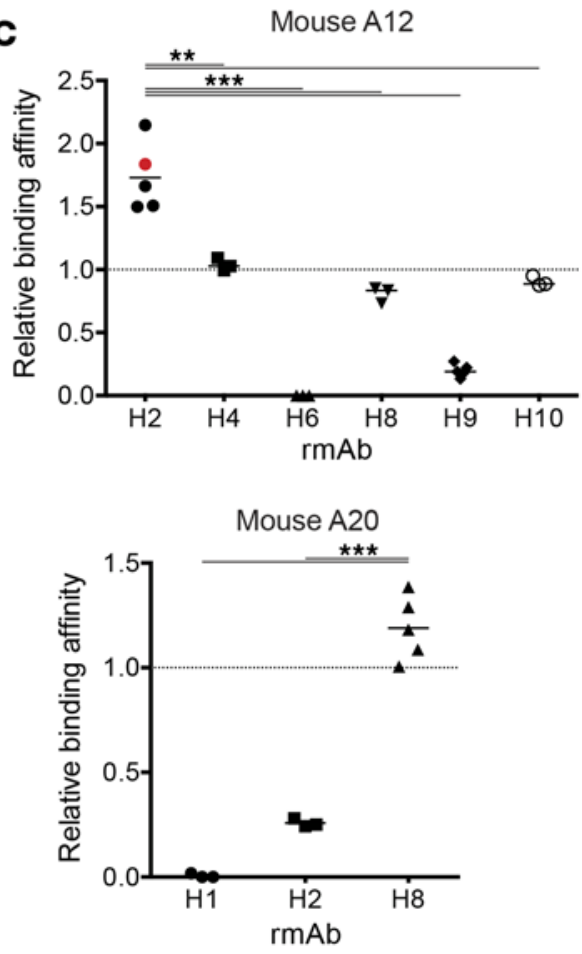

Figure 8. Somatic IgG-VH mutations occurring in mELT cause significant alterations in the binding affinity of the corresponding rmAb to the antigen MOG. (A) Amino acid alignment of the CDR1-CDR3 regions of 9 mutated IgG-VH sequences found in mELT exclusively with the germline sequence (knockin Ig-VH derived from the 8.18-c5 hybridoma). Six mutated sequences are from mouse A12 and 3 from mouse A20. AA position labeling according to IMGT. Dashes indicate consensus with the germline. (B and C) The 9 mutated Ig-VH sequences were coexpressed with the light chain of the 8.18-c5 hybridoma. Non-mutated rm8.18-c5 rmAb served as reference rmAb. Biolayer interferometry (Octet RED384) was performed to measure binding affinity to MOG. (B) Exemplary association and dissociation diagrams of one mutated rmAb (A12-H2) and the reference rmAb (rm8.18- $\mathrm{c5}$ ) to MOC in a dilution series. Dark lines, raw data; red lines, fitted curves. Based on association (on) and dissociation (off) rates for each rmAb and the reference rmAb measured in the same experiment, $K_{D} s$ were determined. rmAb A12-H2 (top): $K_{\text {on }}=1.59 \mathrm{E}+05(1 / \mathrm{ms})( \pm 9.10 \mathrm{E}+02), K_{\text {off }}=1.59 \mathrm{E}-04(1 / \mathrm{s})( \pm 4.24 \mathrm{E}-06), r^{2}>0.99 ; K_{D}=9.97 \mathrm{E}-10$ (M). rm8.18-c5 (bottom): $K_{\text {on }}=2.64 \mathrm{E}+05(1 / \mathrm{Ms})( \pm 1.85 \mathrm{E}+03), K_{\text {off }}=4.82 \mathrm{E}-04(1 / \mathrm{s})( \pm 6.35 \mathrm{E}-06), r^{2}>0.99, K_{D}=1.83 \mathrm{E}-9$ (M). (C) The ratio $K_{D}^{\text {rm8.18-c5 }} / K_{D}^{\text {mut rmab }}$ (designated relative binding affinity) is shown for all 9 mutated rmAb, for each mouse separately. For each pair of mutated rmAb and its reference rmAb, data points of 3-5 independent experiments and the mean are plotted. The data point in red represents the rmAb pair depicted in $\mathbf{B}$. ${ }^{* *} P \leq 0.01 ;{ }^{* * *} P \leq$ 0.001; unpaired 2-tailed $t$ test with Welch's correction (comparing the rmAb with the highest binding affinity with all others). $\mathrm{VH}$, heavy chain variable region; mELT, meningeal ectopic lymphoid tissue; rmAb, recombinant monoclonal Ab; MOG, myelin oligodendrocyte glycoprotein; CDR, complementarity determining region; IMGT, International ImMunoGeneTics.

Software (all Applied Biosystems). Five to 10 targeted areas from the same mELT structure from adjacent slices were combined on one cap for joint RNA isolation. In 3 of the 5 mice, we were able to obtain tissue from more than one contiguous mELT on separate caps (designated mELT 1,2 , and 3 , respectively). If one specific cap was used for analysis, the respective figure is labeled accordingly (e.g., "mELT1"). If all mELT caps from one mouse were analyzed jointly or when mean data from all mice are shown, the figure is labeled "mELT." The total area $\left(\mu \mathrm{m}^{2}\right)$ of captured tissue per cap was recorded, and pictures from each targeted structure were taken before and after capture for documentation. Captured tissue on caps was collected in RLT buffer (QIAGEN) with 1\% 2-mercaptoethanol and carrier RNA in GeneAmp tubes (Applied Biosystems), followed by immediate RNA isolation. 
Preparation of Ig-VH libraries for RepSeq. Total RNA was isolated from fresh single-cell suspensions from the blood, spleen, and LN using the RNeasy Mini Kit including DNase digestion (QIAGEN) according to the manufacturer's instructions. RNA concentration was measured with the NanoDrop 1000 Spectrophotometer (Thermo Scientific), and equal RNA amounts were transcribed into cDNA with the iScript cDNA Synthesis Kit (Bio-Rad), which contains a mix of oligo(dT) and random hexamer primers. Total RNA from fresh-frozen laser-captured mELT was isolated using the RNeasy Micro Kit including DNase digestion (QIAGEN) according to the manufacturer's instructions, and cDNA was synthesized from each sample using the iScript cDNA Synthesis Kit. PCR was performed by amplifying the hypervariable region (including CDR1-CDR3) of the IG heavy chain transcripts present in the respective samples. The primers for the Ig-VH transcripts were designed to amplify a portion within the FR1, starting just upstream of the CDR1 and ending approximately $50 \mathrm{bp}$ downstream of the 5 '-end of the constant region. The forward primer was specific for a region in the FR1 of the $\mathrm{IgH}^{\mathrm{MOG}}$ knockin allele (GenBank AF042086.1), and the reverse primers were specific for either the murine IGHM gene (IgM reverse primer) or the various murine IgG subclasses together (IGHG1, IGHG2A/C, IGHG2B, IGHG3) (degenerate IgG reverse primer). All primers contained adapter sequences for Ion Torrent semiconductor sequencing, and reverse primers contained nucleotide bar code sequences for sample multiplexing on sequencing chips. Forward primer was 5'-CCTCTCTATGGGCAGTCGGTGATCCTCAGTGGAGATATCCTGCAA-3'; IgM reverse primer, 5'-CCATCTCATCCCTGCGTGTCTCCGACTCAG-bar code sequence-ACGAGGGGGAAGACATTTGGGAA-3'; IgG reverse primer, 5'-CCATCTCATCCCTGCGTGTCTCCGACTCAG-bar code sequence-CCAGGGRCCARKGGATARACNGRTGG-3'. The Advantage 2 PCR Kit (Clontech) was used. PCR conditions were (i) $95^{\circ} \mathrm{C}, 60$ seconds; (ii) $95^{\circ} \mathrm{C}, 30$ seconds; $65.9^{\circ} \mathrm{C}$, 30 seconds; $72^{\circ} \mathrm{C}, 60$ seconds ( 33 or 45 cycles); (iii) $72^{\circ} \mathrm{C}, 7$ minutes. PCR products were run on a $1.5 \%$ agarose gel, and the specific band was cut using a Dark Reader blue light transilluminator (Clare Chemical Research). Gel extraction was performed with the QIAquick Gel Extraction Kit (QIAGEN). cDNA was eluted in $30 \mu 1$ $\mathrm{H}_{2} \mathrm{O}$. DNA fragment size, purity, and concentration were determined using the Agilent 2100 Bioanalyzer with the Agilent High Sensitivity DNA Kit (both Agilent Technologies). Samples were diluted to 15 pM, and equimolar amounts of samples were mixed to create cDNA libraries. In general, all samples from one mouse were combined in one library and later sequenced on one chip (one sequencing run). cDNA libraries were stored at $-20^{\circ} \mathrm{C}$ until sequencing.

Ig-VH RepSeq. We used Ion Torrent semiconductor sequencing (Personal Genome Machine [PGM]; Life Technologies) to interrogate the Ig repertoire in this study. As this technology had not been previously used in our hands for Ig RepSeq in a murine model with a single relevant Ig-VH germline segment, we first sought to evaluate the potential influence of this technology on analyses that rely heavily on detection of mutations and can thus be compromised by sequencing errors. In general, next-generation sequencing is potentially error prone, and the sequencing platform used in this study can result in insertion/deletion errors and less frequently in single nucleotide substitution errors. Prior to sequencing Ig-VH libraries from our experimental animals, we performed PCR and sequencing of a plasmid containing a known IgG$\mathrm{VH}$ sequence to calculate the rate of substitution errors (Supplemental Figure 2). The error rate for the sequenced 290-bp region of the plasmid was determined to be 0.087 per read. This number was used to infer the probability that one or more apparent nucleotide substitutions were due to sequencing errors rather than SHM in our Ig-VH cDNA libraries. The probability for a single substitution error to occur in an entire Ig-VH sequencing read of approximately 240 nucleotides is around $8 \%$. Two errors will occur with a $0.35 \%$ probability, 3 errors with a $0.01 \%$ probability, etc. (Supplemental Table 2). Thus, substitution errors are rare overall and are expected to confound SHM analyses only to a minor degree. We then proceeded with sequencing murine Ig-VH libraries. First, cDNA from each library was subjected to emulsion-PCR to link clonally amplified DNA with Ion Sphere Particles (ISP) using the Ion PGM Template OT2 400 Kit in conjunction with the Ion OneTouch 2 System. The percentage of templated ISP was routinely checked using the Ion Sphere Quality Control Kit before enrichment, aiming for 10\%-30\% of templated ISP. Next-generation sequencing of the samples was performed on the Ion PGM System according to the manufacturer's instructions using the Ion PGM Hi-Q Sequencing Kit and Ion 314v2, 316v2, or 318v2 chips, respectively. All instruments, supplies, and kits were from Life Technologies.

Statistics and bioinformatics analysis. All raw sequencing reads were processed using a pipeline adapted from the AbMining tool (42) to determine their CDR3 aa sequences. Subsequently, a BLAST (https://blast.ncbi. nlm.nih.gov/Blast.cgi) search for sequences with identified CDR3s was performed against a custom-made database comprising the knockin Ig-VH sequence as well as all other known mouse IGHV germline genes to 
determine the IGHV gene segment. Only sequences aligning to the knockin Ig-VH $(\geq 99.5 \%)$ were extracted and corrected for insertions and deletions using a pipeline developed in house based on their alignment with the knockin sequence (germline) (Table 1 lists the number of all processed reads). To calculate SHM profiles, sequencing reads with identical CDR1 to CDR3 nucleotide sequences were grouped as nonredundant (unique) reads, and SHMs were quantified for this entire region. Amino acid positions and location of the CDR regions were determined using International ImMunoGeneTics (IMGT)/V-QUEST (43). To generate Kabat-Wu plots, the cumulative mutation frequency was counted for each aa position iteratively for each compartment in the full range of the CDR1 to CDR3 region only for reads covering this entire domain. To assess whether aa replacement mutations were overrepresented in CDRs versus in FRs, mutation counts were summed for the CDRs (CDR1, CDR2, and CDR3 together) and the FRs (FR2 and FR3 together) and normalized for their respective length. A paired 2-tailed $t$ test followed by adjustment for multiple comparisons using the Benjamini-Hochberg method was used to calculate the statistical significance. To assess the overlap of the B cell repertoires of different tissues, unique nucleotide sequences of the entire CDR1 to CDR3 region were extracted from each compartment and compared between all possible pairwise combinations. The quantification was subsequently plotted as Venn diagrams using R version 3.0.2 software (https:// www.r-project.org/) with the VennDiagram package. Ig lineage trees were generated as previously reported $(24,26)$ using IgTree software (provided by R. Mehr, Bar-Ilan University, Ramat-Gan, Israel) (30). Briefly, automated multiple alignments of all Ig-VH sequences from CDR1 to CDR3 with at least 3, 5, or 20 counts, respectively, and the corresponding germline sequence (knockin Ig-VH) were performed using Clustal W 2.1 (44). Lineage trees were displayed in Cytoscape version 2.8.3 (45) using the proprietary organic layout, which permits a more compact depiction of lineages; tree nodes were colored according to their compartment origin and isotype (IgM or IgG). In some instances with excessively large trees, representative fractions thereof are shown. To determine the composition of a constituting the CDR3 at each of its 13 positions, unique CDR3 sequences were extracted from each compartment and CDR3 logos were generated using the WebLogo3 website (http://weblogo.threeplusone.com) (46). To assess the statistical significance of the frequency of specific aa replacements at certain positions in different tissues, the permutation test based on 9999 Monte-Carlo resamplings as calculated in $\mathrm{R}$ with the "Coin" package was utilized. IgG subclass usage was determined by performing a BLAST search of all reads against a custom-made database comprising the germline nucleotide sequences of the murine IgG1, IgG2a/c, IgG2b, and IgG3 constant regions. Sequences with ambiguous hits were excluded from the quantification. To assess the probability of sequencing errors confounding our results, sequencing data derived from an Ig-VH plasmid with a known sequence was processed using the same pipeline as described above. The probability of sequencing errors resulting in false mutations or false nodes in Ig trees was modeled as a Poisson process in R. Prism software v6.0b (GraphPad software) was used for all other statistics analysis not specifically mentioned above. Statistical analysis methods were chosen for each data set to meet the assumptions of the respective tests. A value of $P \leq 0.05$ was considered significant. In general, all experiments were performed once for each of the 5 mice, and all data were included in the analyses, except for IgM-VH analyses, for which data were available only for a subset of mice, or unless otherwise indicated in the respective figure or legend. All next-generation sequencing data were deposited in the NCBI Sequence Read Archive (SRA) (http://www.ncbi.nlm.nih.gov/Traces/sra/; accession no. SRP078959). Computer code other than the software packages described above is available at https://github.com/wszcas/Mouse_EAE.git.

Quantitative PCR of AICDA. Th×2D2 EAE mice and healthy WT mice were perfused with PBS; cubes of spinal cord (including meninges), spleen, and kidney (approximately $2 \mathrm{~mm} \times 2 \mathrm{~mm} \times 2 \mathrm{~mm}$ ) were dissected and immediately frozen on dry ice. Initially, we used quantitative PCR (qPCR) to test tissue collected by LCM, but the AICDA signal was below the limit of detection of the assay, in some instances even in the positive control. We thus modified the protocol to increase the overall RNA amounts by performing RT and qPCR on an approximately $2-\mathrm{mm}^{3}$ piece of thoracic spinal cord including the meninges and any cellular aggregates associated with them. Similarly sized tissue pieces from spleen and kidney were examined. After dissection of all tissues was complete, samples proceeded directly to RNA isolation. Cubes were minced and collected in RLT buffer (QIAGEN) with 1\% 2-mercaptoethanol. Total RNA was extracted using the RNeasy Mini Kit including DNase digestion (QIAGEN) according to the manufacturer's instructions. RNA concentration was measured with the NanoDrop 1000 Spectrophotometer (Thermo Scientific). During establishment of the protocol, RNA integrity was confirmed with the Agilent 2100 Bioanalyzer with the Agilent RNA 6000 Nano Kit (Agilent Technologies). For each mouse equal RNA amounts from all of the respective tissues (for each mouse the maximum amount of RNA available was used; range between mice: 300-2,000 ng) were transcribed 
into cDNA with the iScript cDNA Synthesis Kit (Bio-Rad). DNA contamination was routinely tested with a non-reverse transcriptase control in all assays. AICDA expression was measured using a commercial assay (Mm01184115_m1; Invitrogen; 81-bp amplicon; FAM-MGB). Three reference genes were tested, but only one ( $\beta$-actin [ACTB]; Mm00607939_s1; Invitrogen; 115 bp amplicon; FAM-MGB) was equally expressed in all relevant tissues and was hence used as a reference gene. All commercial assays were validated in our laboratory in compliance with Minimum Information for Publication of Quantitative Real-Time PCR Experiments (MIQE) guidelines $(47,48)$. Specifically, a serial dilution was measured and a calibration curve was generated, testing for PCR efficiency and inhibition and evaluating the limit of detection (LOD), linear dynamic range, and $r^{2}$. qPCR reactions (reaction volume $50 \mu$ l) were performed with the TaqMan Gene Expression Master Mix (cata$\log 4369016$ ) using the 7500 Real Time PCR System and 7500 Software v2.0.6 (all Applied Biosystems). PCR conditions were (i) $50.0^{\circ} \mathrm{C}, 2$ minutes; (b) $95^{\circ} \mathrm{C}, 10$ minutes; (iii) $95^{\circ} \mathrm{C}, 15$ seconds; $60^{\circ} \mathrm{C}, 60$ seconds (50 cycles). Relative expression was calculated using the $\Delta \Delta$ quantification cycle $\left(\mathrm{C}_{\mathrm{q}}\right)$ method (49).

Immunohistochemistry. Three Th $\times 2 \mathrm{D} 2 \mathrm{EAE}$ mice were perfused with and then placed in $10 \%$ neutral-buffered formalin over night. After dissection, spinal cords including the meninges were embedded in paraffin and sectioned (5- $\mu \mathrm{m}$ thickness). After heat-mediated antigen retrieval (in citrate buffer, $\mathrm{pH} 6$ ) and treatment with $0.3 \%$ hydrogen peroxide solution (Sigma-Aldrich), samples were blocked with normal serum $(2.5 \%$ of rabbit or $10 \%$ of rat serum, respectively) and a streptavidin/biotin blocking kit (Vector Laboratories). Standard avidin-biotin immunohistochemical staining was performed on the sections with rabbit anti-mouse CD3 (EPR4517; 1:1,000 dilution; Abcam), rat anti-mouse B220 (CD45R) (RA3-6B2; 1:200 dilution; BD), and rat anti-mouse AICDA (mAID-2; 1:25 dilution; Abcam). Secondary antibodies were anti-rat Ig HRP Detection Kit (BD) in a 1:100 dilution and ImmPRESS HRP anti-Rabbit Ig Polymer Detection Kit (Vector Laboratories), respectively. Slides were counterstained with hematoxylin. Normal mouse spleen tissue served as a positive staining control. Negative controls with omission of the primary antibody were performed routinely. Images were acquired with an Axio Imager M2 with Apotome microscope (objective: 20×/0.8), AxioCam MRm camera, and ZEN (Blue) software (all Zeiss).

Cloning of $r m A b$. Mutated Ig-VH sequences selected from our RepSeq dataset and the Ig light chain (IgL) VJ sequence from the 8.18-c5 hybridoma $\left(\operatorname{IgK}_{\mathrm{V} 8-28}, \operatorname{IgK}_{\mathrm{J} 5}\right)$ were synthesized by GenScript and provided in pUC57 vectors. These were subcloned into eukaryotic expression vectors containing human IgG1 or IgK constant regions (50). Expi293 cells (Life Technologies) were cotransfected with $20 \mu \mathrm{g}$ Ig-VH and $17 \mu \mathrm{g}$ IgL according to the manufacturer's instructions. After 5-6 days, the rmAbs were purified from the supernatants using Protein G beads (GE Healthcare), followed by a PBS buffer exchange (Zeba Spin Desalting Columns, Thermo Scientific). Protein concentrations were determined using the BCA protein assay (Thermo Scientific). Similarly, non-mutated humanized 8.18-c5 rmAb (rm8.18-c5) was produced to serve as a reference. The use of the human $\mathrm{Fc}_{\mathrm{c}}$ region has no effect on the antigen-binding domain and thus does not influence the results of the binding studies.

Antibody binding studies. For biolayer interferometry, various rmAb (ligands) were loaded onto equilibrated anti-human Fc capture biosensors tips (FortéBIO) at a concentration of $200 \mathrm{nM}$ until loading reached $\sim 1.6$ $\mathrm{nm}$. Immobilized $\mathrm{rmAb}$ were then used for recording of association and dissociation sensorgram traces with variable antigen concentrations. The antigen (analyte) recombinant mouse $\mathrm{MOG}_{1-117}$ (mMOG) was added in a 7-step 1:1.2 dilution series, starting with $600 \mathrm{nM}$ as the highest concentration. mMOG was a gift of C.C.A. Bernard (Monash University, Clayton, Victoria, Australia). Binding resulted in a wavelength shift $R_{\max }$ of $\sim 0.3 \mathrm{~nm}$. All measurements were carried out in $\mathrm{PBS}$ at $26^{\circ} \mathrm{C}$ and at $1,000 \mathrm{rpm}$ in 30 -second association and 4-minute dissociation steps with the Octet RED384 platform and software (both FortéBIO). Sensorgrams were aligned to the same baseline after subtraction of the reference measurement (without antigen). After global (full) curve fitting, $r^{2}$ values were $>0.99$. Background binding of $\mathrm{mMOG}$ to the biosensors was tested and found to be negligible. $K_{D}$ values were calculated by application of the following 1:1 binding model: $K_{D}$ $=K_{o f f} / K_{o n}$, where $K_{D}$ is the dissociation constant, $K_{\text {off }}$ is the dissociation rate constant (off rate), and $K_{o n}$ is the association rate constant (on rate). All rmAb were tested 3-5 times each in independent experiments. The non-mutated $\mathrm{rmAb}$ rm8.18-c5 served as an internal reference $\mathrm{rmAb}$, as it was measured together with each of the mutated rmAbs in every experiment under identical conditions. Hence, the ratio $K_{D}{ }^{r m 8.18-c 5} / K_{D}{ }^{m u t r m A b}$ (designated relative binding affinity) was calculated for each mutated rmAb.

Study approval. This study was approved by the UCSF Institutional Animal Care and Use Committee and was in accordance with the United States Public Health Service's Policy on Humane Care and Use of Laboratory Animals. 


\section{Author contributions}

$\mathrm{KLH}, \mathrm{SSZ}$, and HCvB designed the study and wrote the manuscript. KLH and SAS performed experiments. SW performed all bioinformatics analyses. SW and SAS assisted in writing the manuscript. KLH and SW performed statistical analyses. SSZ and HCvB supervised the study.

\section{Acknowledgments}

This work was supported by grants from the Deutsche Forschungsgemeinschaft (DFG; Le 3079/1-1 to KLH), the National Multiple Sclerosis Society (FG 2067-A-1 to KLH; RG4856 to HCvB; RG 4124, RG 4786, and RG 5180 to SSZ), the NIH/National Institute of Neurological Disorders and Stroke (NINDS) (K02NS072288 and R01NS092835 to HCvB; R01AI073737 to SSZ), the Rachleff Family Foundation (HCvB), the Maisin Foundation (SSZ), and the Guthy-Jackson Charitable Foundation (SSZ). The authors would like to thank Fengrong Yan and Montana Horwitz of the Histology Core of the Gladstone Institutes for performing immunohistochemistry, C.C.A. Bernard for providing mMOG, C.M. Spencer for helpful discussion, and Peter Hwang for helpful advice regarding operation of the Octet RED384 instrument.

Address correspondence to: Scott S. Zamvil or H.-Christian von Büdingen, Department of Neurology, University of California, San Francisco, 675 Nelson Rising Lane, NS-215A, San Francisco, California 94158, USA. Phone: 415.502.7395; E-mail: zamvil@ucsf.neuroimmunol.org (S.S. Zamvil); hans-christian. vonbuedingen@ucsf.edu (H.C. von Büdingen).

KLH's current address is: Department of Neurology, Klinikum rechts der Isar, Technische Universität München; Munich Cluster for Systems Neurology (SyNergy), Munich, Germany.

1. Pitzalis C, Jones GW, Bombardieri M, Jones SA. Ectopic lymphoid-like structures in infection, cancer and autoimmunity. Nat Rev Immunol. 2014;14(7):447-462.

2. Weyand CM, Kurtin PJ, Goronzy JJ. Ectopic lymphoid organogenesis: a fast track for autoimmunity. Am J Pathol. 2001;159(3):787-793.

3. Humby F, et al. Ectopic lymphoid structures support ongoing production of class-switched autoantibodies in rheumatoid synovium. PLoS Med. 2009;6(1):e1.

4. Stott DI, Hiepe F, Hummel M, Steinhauser G, Berek C. Antigen-driven clonal proliferation of B cells within the target tissue of an autoimmune disease. The salivary glands of patients with Sjögren's syndrome. J Clin Invest. 1998;102(5):938-946.

5. Serafini B, et al. Dysregulated Epstein-Barr virus infection in the multiple sclerosis brain. J Exp Med. 2007;204(12):2899-2912.

6. Corcione A, et al. Recapitulation of B cell differentiation in the central nervous system of patients with multiple sclerosis. Proc Natl Acad Sci USA. 2004;101(30):11064-11069.

7. Ghosh S, Steere AC, Stollar BD, Huber BT. In situ diversification of the antibody repertoire in chronic Lyme arthritis synovium. J Immunol. 2005;174(5):2860-2869.

8. Nacionales DC, et al. B cell proliferation, somatic hypermutation, class switch recombination, and autoantibody production in ectopic lymphoid tissue in murine lupus. J Immunol. 2009;182(7):4226-4236.

9. Chang A, et al. In situ B cell-mediated immune responses and tubulointerstitial inflammation in human lupus nephritis. J Immunol. 2011;186(3):1849-1860.

10. Scheel T, Gursche A, Zacher J, Häup1 T, Berek C. V-region gene analysis of locally defined synovial B and plasma cells reveals selected B cell expansion and accumulation of plasma cell clones in rheumatoid arthritis. Arthritis Rheum. 2011;63(1):63-72.

11. Nzula S, Going JJ, Stott DI. Antigen-driven clonal proliferation, somatic hypermutation, and selection of B lymphocytes infiltrating human ductal breast carcinomas. Cancer Res. 2003;63(12):3275-3280.

12. Howell OW, et al. Meningeal inflammation is widespread and linked to cortical pathology in multiple sclerosis. Brain. 2011;134(Pt 9):2755-2771.

13. Magliozzi R, et al. A Gradient of neuronal loss and meningeal inflammation in multiple sclerosis. Ann Neurol. 2010;68(4):477493.

14. Serafini B, Rosicarelli B, Magliozzi R, Stigliano E, Aloisi F. Detection of ectopic B-cell follicles with germinal centers in the meninges of patients with secondary progressive multiple sclerosis. Brain Pathol. 2004;14(2):164-174.

15. Haugen M, Frederiksen JL, Degn M. B cell follicle-like structures in multiple sclerosis-with focus on the role of B cell activating factor. J Neuroimmunol. 2014;273(1-2):1-7.

16. Uccelli A, Aloisi F, Pistoia V. Unveiling the enigma of the CNS as a B-cell fostering environment. Trends Immunol. 2005;26(5):254-259

17. Magliozzi R, Columba-Cabezas S, Serafini B, Aloisi F. Intracerebral expression of CXCL13 and BAFF is accompanied by formation of lymphoid follicle-like structures in the meninges of mice with relapsing experimental autoimmune encephalomyelitis. J Neuroimmunol. 2004;148(1-2):11-23.

18. Columba-Cabezas S, et al. Suppression of established experimental autoimmune encephalomyelitis and formation of meningeal lymphoid follicles by lymphotoxin beta receptor-Ig fusion protein. J Neuroimmunol. 2006;179(1-2):76-86.

19. Peters A, et al. Th17 cells induce ectopic lymphoid follicles in central nervous system tissue inflammation. Immunity. 
2011;35(6):986-996

20. Molnarfi N, et al. MHC class II-dependent B cell APC function is required for induction of CNS autoimmunity independent of myelin-specific antibodies. J Exp Med. 2013;210(13):2921-2937.

21. Bettelli E, Baeten D, Jäger A, Sobel RA, Kuchroo VK. Myelin oligodendrocyte glycoprotein-specific T and B cells cooperate to induce a Devic-like disease in mice. J Clin Invest. 2006;116(9):2393-2402.

22. Krishnamoorthy G, Lassmann H, Wekerle H, Holz A. Spontaneous opticospinal encephalomyelitis in a double-transgenic mouse model of autoimmune T cell/B cell cooperation. J Clin Invest. 2006;116(9):2385-2392.

23. Linnington C, Webb M, Woodhams PL. A novel myelin-associated glycoprotein defined by a mouse monoclonal antibody. $J$ Neuroimmunol. 1984;6(6):387-396.

24. Palanichamy A, et al. Immunoglobulin class-switched B cells form an active immune axis between CNS and periphery in multiple sclerosis. Sci Transl Med. 2014;6(248):248ra106.

25. Stern JN, et al. B cells populating the multiple sclerosis brain mature in the draining cervical lymph nodes. Sci Transl Med. 2014;6(248):248ra107.

26. von Büdingen HC, et al. B cell exchange across the blood-brain barrier in multiple sclerosis. J Clin Invest. 2012;122(12):4533-4543

27. Bose B, Sinha S. Problems in using statistical analysis of replacement and silent mutations in antibody genes for determining antigen-driven affinity selection. Immunology. 2005;116(2):172-183.

28. Wu TT, Kabat EA. An analysis of the sequences of the variable regions of Bence Jones proteins and myeloma light chains and their implications for antibody complementarity. J Exp Med. 1970;132(2):211-250.

29. Margolin DH, et al. Germinal center function in the spleen during simian HIV infection in rhesus monkeys. J Immunol. 2006;177(2):1108-1119.

30. Barak M, Zuckerman NS, Edelman H, Unger R, Mehr R. IgTree: creating immunoglobulin variable region gene lineage trees. $J$ Immunol Methods. 2008;338(1-2):67-74

31. Lovato L, et al. Related B cell clones populate the meninges and parenchyma of patients with multiple sclerosis. Brain. 2011;134(Pt 2):534-541.

32. Breithaupt C, et al. Structural insights into the antigenicity of myelin oligodendrocyte glycoprotein. Proc Natl Acad Sci U S A. 2003;100(16):9446-9451.

33. Litzenburger T, et al. B lymphocytes producing demyelinating autoantibodies: development and function in gene-targeted transgenic mice. J Exp Med. 1998;188(1):169-180.

34. Litzenburger T, et al. Development of myelin oligodendrocyte glycoprotein autoreactive transgenic B lymphocytes: receptor editing in vivo after encounter of a self-antigen distinct from myelin oligodendrocyte glycoprotein. J Immunol. 2000;165(9):5360-5366.

35. Lanzavecchia A. Antigen-specific interaction between T and B cells. Nature. 1985;314(6011):537-539.

36. Constant S, Schweitzer N, West J, Ranney P, Bottomly K. B lymphocytes can be competent antigen-presenting cells for priming CD4+ T cells to protein antigens in vivo. J Immunol. 1995;155(8):3734-3741.

37. Hauser SL, et al. B-cell depletion with rituximab in relapsing-remitting multiple sclerosis. N Engl J Med. 2008;358(7):676-688.

38. Kappos L, et al. Ocrelizumab in relapsing-remitting multiple sclerosis: a phase 2, randomised, placebo-controlled, multicentre trial. Lancet. 2011;378(9805):1779-1787.

39. Thaunat $\mathrm{O}$, et al. B cell survival in intragraft tertiary lymphoid organs after rituximab therapy. Transplantation. 2008;85(11):1648-1653.

40. Gong Q, et al. Importance of cellular microenvironment and circulatory dynamics in B cell immunotherapy. J Immunol. 2005;174(2):817-826

41. Bettelli E, Pagany M, Weiner HL, Linington C, Sobel RA, Kuchroo VK. Myelin oligodendrocyte glycoprotein-specific T cell receptor transgenic mice develop spontaneous autoimmune optic neuritis. J Exp Med. 2003;197(9):1073-1081.

42. D'Angelo S, et al. The antibody mining toolbox: an open source tool for the rapid analysis of antibody repertoires. $M A b s$. 2014;6(1):160-172.

43. Brochet X, Lefranc MP, Giudicelli V. IMGT/V-QUEST: the highly customized and integrated system for IG and TR standardized V-J and V-D-J sequence analysis. Nucleic Acids Res. 2008;36(Web Server issue):W503-W508.

44. Larkin MA, et al. Clustal W and Clustal X version 2.0. Bioinformatics. 2007;23(21):2947-2948.

45. Smoot ME, Ono K, Ruscheinski J, Wang PL, Ideker T. Cytoscape 2.8: new features for data integration and network visualization. Bioinformatics. 2011;27(3):431-432.

46. Crooks GE, Hon G, Chandonia JM, Brenner SE. WebLogo: a sequence logo generator. Genome Res. 2004;14(6):1188-1190.

47. Bustin SA, et al. The MIQE guidelines: minimum information for publication of quantitative real-time PCR experiments. Clin Chem. 2009;55(4):611-622.

48. Bustin SA, et al. Primer sequence disclosure: a clarification of the MIQE guidelines. Clin Chem. 2011;57(6):919-921.

49. Livak KJ, Schmittgen TD. Analysis of relative gene expression data using real-time quantitative PCR and the 2(-Delta Delta C(T)) Method. Methods. 2001;25(4):402-408

50. Tiller T, Meffre E, Yurasov S, Tsuiji M, Nussenzweig MC, Wardemann H. Efficient generation of monoclonal antibodies from single human B cells by single cell RT-PCR and expression vector cloning. J Immunol Methods. 2008;329(1-2):112-124 